\title{
Superintegrability and associated polynomial solutions: Euclidean space and the sphere in two dimensions
}

\author{
E. G. Kalnins \\ Department of Mathematics and Statistics, University of Waikato, Hamilton, New Zealand \\ W. Miller, Jr. \\ School of Mathematics, University of Minnesota, Minneapolis, Minnesota 55455
}

G. S. Pogosyan

Laboratory of Theoretical Physics, Joint Institute for Nuclear Research, Dubna, Moscow Region 141980, Russia

(Received 1 May 1996; accepted for publication 7 August 1996)

\begin{abstract}
In this work we examine the basis functions for those classical and quantum mechanical systems in two dimensions which admit separation of variables in at least two coordinate systems. We do this for the corresponding systems defined in Euclidean space and on the two-dimensional sphere. We present all of these cases from a unified point of view. In particular, all of the special functions that arise via variable separation have their essential features expressed in terms of their zeros. The principal new results are the details of the polynomial bases for each of the nonsubgroup bases, not just the subgroup Cartesian and polar coordinate cases, and the details of the structure of the quadratic algebras. We also study the polynomial eigenfunctions in elliptic coordinates of the $n$-dimensional isotropic quantum oscillator. (C) 1996 American Institute of Physics. [S0022-2488(96)03212-4]
\end{abstract}

\section{INTRODUCTION}

It has long been known that there are potentials for which a given Hamiltonian system in classical mechanics admits a solution via separation of variables in more than one coordinate system. ${ }^{1}$ The methodical search for such potentials was initiated by Smorodinsky and Winternitz et al. in two and three dimensions, ${ }^{2-4}$ and there has been a considerable amount of work for various examples. ${ }^{5-9}$ A subset of such systems is called maximal in dimension $N$ if there exist $2 N-1$ functionally independent integrals of motion. In some papers, such systems are called superintegrable. ${ }^{10,11}$ In this work we examine the basis functions for those classical and quantum mechanical systems in two dimensions which admit separation of variables in at least two coordinate systems. We do this for the corresponding systems defined in Euclidean space and on the two-dimensional sphere. In a subsequent work we shall study systems defined in two-dimensional hyperbolic spaces and in complex two-dimensional spaces.

For each of the superintegrable systems we observe that, for the discrete spectrum of the quantum mechanical Hamiltonian, one can consider this operator as acting on a space of polynomials. ${ }^{12}$ Each eigenvalue is multiply degenerate. However, each separable coordinate system gives rise to an orthonormal basis of polynomial eigenfunctions in this space and breaks the degeneracy. These bases are characterized as simultaneous eigenfunctions of second-order symmetry operators for the Hamiltonian. ${ }^{13}$ We show that under commutation these symmetry operators close to form a quadratic algebra. ${ }^{14}$ The superintegral systems are of two types: the "normal type" in which the original Hamiltonian is diagonalized, and the "conformal type" in which the Hamiltonian is modified by multiplying the eigenvalue equation by a function and considering the energy as fixed. The modified equation is then interpreted as the eigenvalue equation for a Hamiltonian on a conformal Euclidean space with a "charge" as the eigenvalue. We present all of these cases from a unified point of view. In particular, all of the special functions that arise via variable separation have their essential features expressed in terms of their zeros. The principal new results 
are the details of the polynomial bases for each of the nonsubgroup bases, not just the subgroup Cartesian and polar coordinate cases, and the details of the structure of the quadratic algebras. (For those coordinate systems which correspond to subgroup type coordinates, the finite solutions have already been found. The contribution of this article is to indicate how the remaining finite solutions can be obtained. This invariably involves the use of a Niven-type ansatz for the finite solutions; every quantity of interest can be computed in terms of the zeros of the corresponding polynomial solutions.) For the sake of completeness we list all the finite solutions that are possible. We analyze each of the potentials systematically.

In Secs. II and III we consider the superintegrable systems in Euclidean two-space and on the two-dimensional sphere, respectively. In Sec. IV we examine the polynomial eigenfunctions in elliptic coordinates of the $n$-dimensional isotropic quantum oscillator.

\section{TWO-DIMENSIONAL EUCLIDEAN SPACE}

As is well known ${ }^{1,13}$ there are exactly four coordinate systems on the Euclidean plane in which the free particle Schrödinger equation separates: Cartesian, polar, parabolic, and elliptic. We describe these coordinate systems. Let $x$ and $y$ be the Cartesian coordinates. Polar coordinates are defined by

$$
\begin{gathered}
x=r \cos \theta, \quad r>0, \\
y=r \sin \theta, \quad 0 \leqslant \theta<2 \pi .
\end{gathered}
$$

Parabolic coordinates. We can define two mutually perpendicular parabolic systems:

$$
x=\frac{1}{2}\left(\xi^{2}-\eta^{2}\right), \quad y=\xi \eta, \quad \xi \in \mathbf{R}, \eta>0,
$$

and

$$
x=\bar{\xi} \bar{\eta}, \quad y=\frac{1}{2}\left(\bar{\xi}^{2}-\bar{\eta}^{2}\right), \quad \bar{\xi} \in \mathbf{R}, \bar{\eta}>0 .
$$

The connection $(\xi, \eta) \mapsto(\bar{\xi}, \bar{\eta})$ is a rotation of angle $\pi / 4$ in the $(\xi, \eta)$ space. The transformation $(x, y) \mapsto(\xi, \eta)$ actually is the two-dimensional realization of the Levi-Civita transformation ${ }^{15}$ which has the form

$$
x=\xi^{2}-\eta^{2}, \quad y=2 \xi \eta, \quad \xi \in \mathbf{R}, \eta>0 .
$$

Elliptic coordinates in algebraic form are defined by $\left(e_{1}<u_{1}<e_{2}<u_{2}\right)$

$$
x^{2}=\frac{\left(u_{1}-e_{1}\right)\left(u_{2}-e_{1}\right)}{e_{2}-e_{1}}, \quad y^{2}=\frac{\left(u_{1}-e_{2}\right)\left(u_{2}-e_{2}\right)}{e_{1}-e_{2}} .
$$

Replacing $x \mapsto x+\sqrt{e_{2}-e_{1}}$ yields a coordinate system which is called elliptic II coordinates: ${ }^{16}$

$$
x=\sqrt{\frac{\left(u_{1}-e_{1}\right)\left(u_{2}-e_{1}\right)}{e_{2}-e_{1}}}+\sqrt{e_{2}-e_{1}}, \quad y=\sqrt{\frac{\left(u_{1}-e_{2}\right)\left(u_{2}-e_{2}\right)}{e_{1}-e_{2}}} .
$$

It is interesting that the two elliptic coordinates are connected by the Levi-Civita transformation. Indeed, if we use parabolic coordinates $(\xi, \eta)$ in Eq. (4) as Cartesian coordinates in Eq. (5), we have

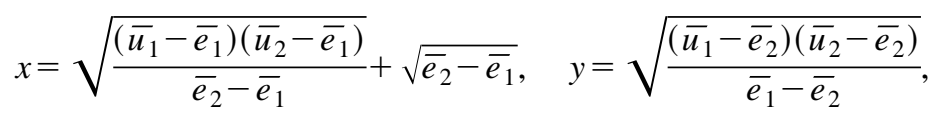


TABLE I. The two-dimensional superintegrable potentials.

\begin{tabular}{ccc}
\hline \hline Potential $V(x, y)$ & $\begin{array}{c}\text { Coordinate } \\
\text { system }\end{array}$ \\
\hline$V_{1}=\frac{1}{2} \omega^{2}\left(x^{2}+y^{2}\right)+\frac{1}{2}\left(\frac{k_{1}^{2}-\frac{1}{4}}{x^{2}}+\frac{k_{2}^{2}-\frac{1}{4}}{y^{2}}\right)$ & Cartesian polar elliptic \\
$V_{2}=\frac{1}{2} \omega^{2}\left(4 x^{2}+y^{2}\right)+k_{1} x+\frac{1}{2} \frac{k_{2}^{2}-\frac{1}{4}}{y^{2}}$ & Cartesian parabolic \\
$V_{3}=$ & $-\frac{\alpha}{\sqrt{x^{2}+y^{2}}+\frac{1}{4} \frac{1}{\sqrt{x^{2}+y^{2}}}}$ & \\
& $\times\left(\frac{k_{1}^{2}-\frac{1}{4}}{\sqrt{x^{2}+y^{2}}+x}+\frac{k_{2}^{2}-\frac{1}{4}}{\sqrt{x^{2}+y^{2}}-x}\right)$ & \\
$V_{4}=$ & $-\frac{\alpha}{\sqrt{x^{2}+y^{2}}+\frac{1}{4} \frac{1}{\sqrt{x^{2}+y^{2}}}}$ & \\
& $\times\left(\beta_{1} \sqrt{\sqrt{x^{2}+y^{2}}+x}+\beta_{2} \sqrt{\sqrt{x^{2}+y^{2}}-x}\right)$ & \\
\hline \hline
\end{tabular}

where

$$
\overline{u_{i}}=u_{i}^{2}-u_{1}\left(e_{1}+e_{2}\right), \quad \overline{e_{1}}=-\frac{1}{4}\left(e_{1}+e_{2}\right)^{2}, \quad \overline{e_{2}}=-e_{1} e_{2} .
$$

As shown in Refs. 2-4 there are four classes of potentials for which multiple separation occurs in Euclidean space (see Table I).

We treat the cases in order. In each case the Schrödinger equation is $(\hbar=m=1)$

$$
-\frac{1}{2} \Delta \Psi+V_{i}(x, y) \Psi=E \Psi, \quad i=1, \ldots, 4 .
$$

(i) The potential in the first case is

$$
V_{1}(x, y)=\frac{1}{2} \omega^{2}\left(x^{2}+y^{2}\right)+\frac{1}{2}\left(\frac{k_{1}^{2}-\frac{1}{4}}{x^{2}}+\frac{k_{2}^{2}-\frac{1}{4}}{y^{2}}\right) .
$$

The corresponding Schrödinger equation is $\left(k_{1}, k_{2}>0\right)$

$$
\left[\frac{\partial^{2}}{\partial x^{2}}+\frac{\partial^{2}}{\partial y^{2}}\right] \Psi+\left[2 E-\omega^{2}\left(x^{2}+y^{2}\right)-\frac{k_{1}^{2}-\frac{1}{4}}{x^{2}}-\frac{k_{2}^{2}-\frac{1}{4}}{y^{2}}\right] \Psi=0 .
$$

See Refs. 2, 3, and 16-18 for an earlier treatment. This equation separates in three different separable coordinate systems: Cartesian, polar, and elliptical coordinates.

If we rewrite the Schrödinger equation by putting

$$
\Psi=e^{-\omega\left(x^{2}+y^{2}\right) / 2} x^{1 / 2 \pm k_{1}} y^{1 / 2 \pm k_{2}} \Phi(x, y),
$$

then the equation for $\Phi(x, y)$ is $Q \Phi=-2 E \Phi$ or 


$$
\left[\frac{\partial^{2}}{\partial x^{2}}+\frac{\partial^{2}}{\partial y^{2}}+\left(\frac{1 \pm 2 k_{1}}{x}-2 \omega x\right) \frac{\partial}{\partial x}+\left(\frac{1 \pm 2 k_{2}}{y^{2}}-2 \omega y\right) \frac{\partial}{\partial y}-2 \omega\left(2 \pm k_{1} \pm k_{2}\right)\right] \Phi=-2 E \Phi .
$$

Clearly the operator $Q$ maps polynomials (in $x^{2}, y^{2}$ ) into polynomials, without increasing the order. Since the original Hamiltonian is self-adjoint with respect to the measure $d x d y$ in the plane, the operator $Q$ acting on polynomials in $x^{2}$ and $y^{2}$ is self-adjoint with respect to the measure

$$
d \rho(x, y)=e^{-\omega\left(x^{2}+y^{2}\right)} x^{1 \pm 2 k_{1}} y^{1 \pm 2 k_{2}} d x d y
$$

in the quadrant $x>0, y>0$. We assume that the positive sign at the $k_{i}$ has to be taken if $k_{i}>\frac{1}{2}$ and both the positive and the negative sign must be taken if $0<k_{i}<\frac{1}{2}$, so that the polynomials have finite norm. ${ }^{16}$

To find the eigenfunctions in the case of Cartesian coordinates we look for separable solutions $\Phi(x, y)=X(x) Y(y)$. Then the separation equation in $x$ becomes

$$
\left[\frac{\partial^{2}}{\partial x^{2}}+\left(-2 \omega+\frac{1 \pm 2 k_{1}}{x^{2}}\right) x \frac{\partial}{\partial x}-\left(1 \pm 2 k_{1}\right) \omega\right] X=2 \lambda_{1} X .
$$

If we look for solutions of the form

$$
X(x)=\prod_{m=1}^{q}\left(x^{2}-\theta_{m}\right)
$$

the $\theta_{m}$ must satisfy

$$
\omega \theta_{\ell}=1 \pm k_{1}+2 \sum_{j \neq \ell} \frac{\theta_{\ell}}{\theta_{\ell}-\theta_{j}}
$$

and

$$
\lambda_{1}=-\omega\left(2 q \pm k_{1}+\frac{1}{2}\right)
$$

where $q$ is a non-negative integer. The solutions are $X(x)=L_{q}^{k_{1}}\left(\omega x^{2}\right)$ where $L_{n}^{\alpha}(z)$ is a Laguerre polynomial.

Thus the corresponding set of orthonormal eigenfunctions which are normalized in quadrant $x>0, y>0$ by

$$
\int_{0}^{\infty} d x \int_{0}^{\infty} d y \Psi_{n_{1}^{\prime} n_{2}^{\prime}}^{*}(x, y) \Psi_{n_{1} n_{2}}(x, y)=\frac{1}{4} \delta_{n_{1}^{\prime} n_{1}} \delta_{n_{2}^{\prime} n_{2}}
$$

is

$$
\begin{aligned}
\Psi(x, y) \equiv & \Psi_{n_{1}, n_{2}}^{\left(k_{1}, k_{2}\right)}(x, y)=\sqrt{\frac{\omega n_{1} ! n_{2} !}{\Gamma\left(n_{1} \pm k_{1}+1\right) \Gamma\left(n_{2} \pm k_{2}+1\right)}}(\sqrt{\omega} x)^{1 / 2 \pm k_{1}}(\sqrt{\omega} y)^{1 / 2 \pm k_{2}} \\
& \times e^{-(\omega / 2)\left(x^{2}+y^{2}\right)} L_{n_{1}}^{ \pm k_{1}}\left(\omega x^{2}\right) L_{n_{2}}^{ \pm k_{2}}\left(\omega y^{2}\right),
\end{aligned}
$$

where $E_{n}=\omega\left(2 n \pm k_{1} \pm k_{2}+2\right)$ and $n=n_{1}+n_{2}$ is the principal quantum number.

The corresponding classical operator is 


$$
L_{1}=p_{x}^{2}-\left(\omega^{2} x^{2}+\frac{k_{1}^{2}-\frac{1}{4}}{x^{2}}\right)
$$

These eigenfunctions satisfy

$$
\hat{L}_{1} \Psi=\left(\frac{\partial^{2}}{\partial x^{2}}-\omega^{2} x^{2}-\frac{k_{1}^{2}-\frac{1}{4}}{x^{2}}\right) \Psi=2 \lambda_{1} \Psi
$$

In polar coordinates the Schrödinger equation is

$$
\left(\frac{\partial^{2}}{\partial r^{2}}+\frac{1}{r} \frac{\partial}{\partial r}+\frac{1}{r^{2}} \frac{\partial^{2}}{\partial \theta^{2}}\right) \Psi+\left[2 E-\omega^{2} r^{2} \Psi-\frac{1}{r^{2}}\left(\frac{k_{1}^{2}-\frac{1}{4}}{\cos ^{2} \theta}+\frac{k_{2}^{2}-\frac{1}{4}}{\sin ^{2} \theta}\right)\right] \Psi=0 .
$$

If we write $\Psi=R(r) T(\theta)$, then the separation equation for $T(\theta)$ is

$$
\frac{d^{2}}{d \theta^{2}} T(\theta)-\left(\frac{k_{1}^{2}-\frac{1}{4}}{\cos ^{2} \theta}+\frac{k_{2}^{2}-\frac{1}{4}}{\sin ^{2} \theta}\right) T(\theta)=\lambda T(\theta) .
$$

If we now put

$$
T(\theta)=(\cos \theta)^{1 / 2 \pm k_{1}}(\sin \theta)^{1 / 2 \pm k_{2}} P(z),
$$

where $z=\cos 2 \theta$, this separation equation becomes

$$
\left\{\left(1-z^{2}\right) \frac{\partial^{2}}{\partial z^{2}}-\left[\left(2 \pm k_{1} \pm k_{2}\right) z+\left( \pm k_{1} \mp k_{2}\right)\right] \frac{\partial}{\partial z}-\frac{1}{4}\left[\lambda+\left(1 \pm k_{1} \pm k_{2}\right)^{2}\right]\right\} P(z)=0 .
$$

If we try solutions of the form $P(z)=\prod_{m=1}^{q}\left(z-\theta_{m}\right)$, we find that the zeros of the polynomial $P(z)$ satisfy

$$
2 \sum_{m \neq l} \frac{1}{\theta_{\ell}-\theta_{m}}=\frac{1 \pm k_{2}}{\left(1-\theta_{\ell}\right)}-\frac{1 \pm k_{1}}{\left(1+\theta_{\ell}\right)}
$$

where $\lambda=-\left(2 q \pm k_{1} \pm k_{2}+1\right)^{2}$. These are just the equations satisfied by the zeros of the Jacobi polynomials. In fact the solutions of this eigenvalue equation have the form

$$
T(\theta)=(\cos \theta)^{1 / 2 \pm k_{1}}(\sin \theta)^{1 / 2 \pm k_{2}} P_{q}^{\left( \pm k_{1}, \pm k_{2}\right)}(\cos 2 \theta) .
$$

The classical constant of the motion is

$$
L_{2}=\left(x p_{y}-y p_{x}\right)^{2}-\left[\frac{k_{1}^{2}-\frac{1}{4}}{x^{2}}+\frac{k_{2}^{2}-\frac{1}{4}}{y^{2}}\right]\left(x^{2}+y^{2}\right)
$$

and the corresponding eigenvalue equation satisfied by the eigenfunctions $\Psi$ is

$$
\hat{L}_{2} \Psi=\left[\left(x \frac{\partial}{\partial y}-y \frac{\partial}{\partial x}\right)^{2}+\left(\frac{\frac{1}{4}-k_{1}^{2}}{x^{2}}+\frac{\frac{1}{4}-k_{2}^{2}}{y^{2}}\right)\left(x^{2}+y^{2}\right)\right] \Psi=\lambda \Psi .
$$

The orthonormal [as in Eq. (14)] eigenfunctions in these coordinates are 


$$
\begin{aligned}
\Psi(r, \theta) \equiv & \Psi_{n_{r}, q}^{\left( \pm k_{1}, \pm k_{2}\right)}(r, \theta)=\Phi_{q}^{\left( \pm k_{1}, \pm k_{2}\right)}(\theta) \sqrt{\frac{2 \omega n !}{\Gamma\left(n+2 q \pm k_{1} \pm k_{2}+1\right)}} \\
& \times e^{-\omega r^{2} / 2}(\sqrt{\omega} r)^{\left(2 q \pm k_{1} \pm k_{2}+1\right)} L_{n}^{2 q \pm k_{1} \pm k_{2}+1}\left(\omega r^{2}\right),
\end{aligned}
$$

where

$$
\begin{aligned}
\Phi_{q}^{\left( \pm k_{1}, \pm k_{2}\right)}(\theta)= & \sqrt{\frac{\left(2 q \pm k_{1} \pm k_{2}+1\right) q ! \Gamma\left(q \pm k_{1} \pm k_{2}+1\right)}{2 \Gamma\left(q \pm k_{2}+1\right) \Gamma\left(q \pm k_{1}+1\right)}} \\
& \times(\cos \theta)^{1 / 2 \pm k_{1}}(\sin \theta)^{1 / 2 \pm k_{2}} P_{q}^{\left( \pm k_{1}, \pm k_{2}\right)}(\cos 2 \theta)
\end{aligned}
$$

and $E=\omega\left(2 n \pm k_{1} \pm k_{2}+2\right)$, with $n=n_{r}+q$. Using (11) we can easily split off the polynomial portion of (20) from the multiplier.

In elliptical coordinates we adopt a similar approach. Using the identity

$$
\frac{x^{2}}{\theta-e_{1}}+\frac{y^{2}}{\theta-e_{2}}-1=-\frac{\left(u_{1}-\theta\right)\left(u_{2}-\theta\right)}{\left(\theta-e_{1}\right)\left(\theta-e_{2}\right)}
$$

we look for solutions (11) of the form ${ }^{19}$

$$
\Phi(x, y)=\prod_{m=1}^{q}\left(\frac{x^{2}}{\theta_{m}-e_{1}}+\frac{y^{2}}{\theta_{m}-e_{2}}-1\right) .
$$

The $\theta_{m}$ must satisfy

$$
\frac{1 \pm k_{1}}{\theta_{m}-e_{1}}+\frac{1 \pm k_{2}}{\theta_{m}-e_{2}}+\sum_{j \neq m} \frac{2}{\left(\theta_{m}-\theta_{j}\right)}-\omega=0
$$

where $E=\omega\left(2 q \pm k_{1} \pm k_{2}+2\right)$. If we write the Schrödinger equation for this potential in elliptical coordinates we obtain

$$
\begin{aligned}
& \frac{4\left(u_{1}-e_{1}\right)\left(u_{1}-e_{2}\right)}{u_{1}-u_{2}}\left[\frac{\partial^{2} \Psi}{\partial u_{1}^{2}}+\frac{1}{2}\left(\frac{1}{u_{1}-e_{1}}+\frac{1}{u_{1}-e_{2}}\right) \frac{\partial \Psi}{\partial u_{1}}\right] \\
& +\frac{4\left(u_{2}-e_{1}\right)\left(u_{2}-e_{2}\right)}{u_{2}-u_{1}}\left[\frac{\partial^{2} \Psi}{\partial u_{2}^{2}}+\frac{1}{2}\left(\frac{1}{u_{2}-e_{1}}+\frac{1}{u_{2}-e_{2}}\right) \frac{\partial \Psi}{\partial u_{2}}\right] \\
& +\left[2 E-\omega^{2}\left(u_{1}+u_{2}-e_{1}-e_{2}\right)+\frac{\left(\frac{1}{4}-k_{1}^{2}\right)\left(e_{2}-e_{1}\right)}{\left(u_{1}-e_{1}\right)\left(u_{2}-e_{1}\right)}+\frac{\left(\frac{1}{4}-k_{2}^{2}\right)\left(e_{1}-e_{2}\right)}{\left(u_{1}-e_{2}\right)\left(u_{2}-e_{2}\right)}\right] \Psi=0 .
\end{aligned}
$$

This equation admits separable solutions $\Psi=U_{1}\left(u_{1}\right) U_{2}\left(u_{2}\right)$ and separation equations

$$
\begin{gathered}
\left\{4\left(u_{i}-e_{1}\right)\left(u_{i}-e_{2}\right)\left[\frac{\partial^{2}}{\partial u_{i}^{2}}+\frac{1}{2}\left(\frac{1}{u_{i}-e_{1}}+\frac{1}{u_{i}-e_{2}}\right) \frac{\partial}{\partial u_{i}}\right]-\omega^{2}\left[u_{i}^{2}-\left(e_{1}+e_{2}\right) u_{i}\right]\right. \\
\left.+\frac{\left(k_{1}^{2}-\frac{1}{4}\right)\left(e_{1}-e_{2}\right)}{\left(u_{1}-e_{1}\right)}+\frac{\left(k_{2}^{2}-\frac{1}{4}\right)\left(e_{2}-e_{1}\right)}{\left(u_{1}-e_{2}\right)}+2 E u_{i}+\lambda\right\} U_{i}\left(u_{i}\right)=0, \quad i=1,2 .
\end{gathered}
$$

The additional operator describing this coordinate system is 


$$
\begin{aligned}
\hat{L}_{3} \Psi= & \frac{4 u_{2}\left(u_{1}-e_{1}\right)\left(u_{1}-e_{2}\right)}{u_{1}-u_{2}}\left[\frac{\partial^{2} \Psi}{\partial u_{1}^{2}}+\frac{1}{2}\left(\frac{1}{u_{1}-e_{1}}+\frac{1}{u_{1}-e_{2}}\right) \frac{\partial \Psi}{\partial u_{1}}\right] \\
& +\frac{4 u_{1}\left(u_{2}-e_{1}\right)\left(u_{2}-e_{2}\right)}{u_{2}-u_{1}}\left[\frac{\partial^{2} \Psi}{\partial u_{2}^{2}}+\frac{1}{2}\left(\frac{1}{u_{2}-e_{1}}+\frac{1}{u_{2}-e_{2}}\right) \frac{\partial \Psi}{\partial u_{2}}\right] \\
& +\left[-u_{1} u_{2} \omega^{2}+\frac{\left(k_{1}^{2}-\frac{1}{4}\right)\left(e_{1}-e_{2}\right)}{\left(u_{1}-e_{1}\right)\left(u_{2}-e_{1}\right)}+\frac{\left(k_{2}^{2}-\frac{1}{4}\right)\left(e_{1}-e_{2}\right)}{\left(u_{1}-e_{2}\right)\left(u_{2}-e_{2}\right)}\right] \Psi \\
= & {\left[\left(x \frac{\partial}{\partial y}-y \frac{\partial}{\partial x}\right)^{2}+e_{2} \frac{\partial^{2}}{\partial x^{2}}+e_{1} \frac{\partial^{2}}{\partial y^{2}}-\omega^{2}\left(e_{2} x^{2}+e_{1} y^{2}+e_{1} e_{2}\right)\right.} \\
& \left.+\frac{\frac{1}{4}-k_{1}^{2}}{x^{2}}\left(x^{2}+y^{2}+e_{2}\right)+\frac{\frac{1}{4}-k_{2}^{2}}{y^{2}}\left(x^{2}+y^{2}+e_{1}\right)\right] \Psi=\lambda \Psi,
\end{aligned}
$$

where

$$
\lambda=-\left(1 \pm k_{1} \pm k_{2}\right)^{2}-2 e_{2} \omega\left(1 \pm k_{1}\right)-2 e_{1} \omega\left(1 \pm k_{2}\right)-\omega^{2} e_{1} e_{2}-4 \sum_{m=1}^{q}\left[e_{2} \frac{1 \pm k_{1}}{\theta_{m}-e_{1}}+e_{1} \frac{1 \pm k_{2}}{\theta_{m}-e_{2}}\right]
$$

The corresponding classical operator is

$$
\begin{aligned}
L_{3}= & \left(x p_{y}-y p_{x}\right)^{2}+e_{2} p_{x}^{2}+e_{1} p_{y}^{2}-\omega^{2}\left(e_{2} x^{2}+e_{1} y^{2}+e_{1} e_{2}\right) \\
& +\frac{\left(\frac{1}{4}-k_{1}^{2}\right)}{x^{2}}\left(x^{2}+y^{2}+e_{2}\right)+\frac{\left(\frac{1}{4}-k_{2}^{2}\right)}{y^{2}}\left(x^{2}+y^{2}+e_{1}\right) .
\end{aligned}
$$

If we now use the redefined operators

$$
M_{1}=L_{1}, \quad M_{2}=L_{2}-\frac{1}{2}+k_{1}^{2}+k_{2}^{2}, \quad M_{3}=L_{3}-\frac{1}{2}+k_{1}^{2}+k_{2}^{2},
$$

and

$$
H=p_{x}^{2}+p_{y}^{2}-\omega^{2}\left(x^{2}+y^{2}\right)+\frac{\frac{1}{4}-k_{1}^{2}}{x^{2}}+\frac{\frac{1}{4}-k_{2}^{2}}{y^{2}}
$$

we observe the following relations:

$$
\begin{aligned}
& M_{12}=\left\{M_{1}, M_{2}\right\}=4\left(x p_{y}-y p_{x}\right)\left(p_{x} p_{y}-\omega^{2} x y\right)+\frac{\frac{1}{4}-k_{2}^{2}}{y^{2}} x p_{x}-\frac{\frac{1}{4}-k_{1}^{2}}{x^{2}} y p_{y}, \\
& \left\{M_{12}, M_{1}\right\}=8\left(M_{1}^{2}-M_{1} H\right)-16 \omega^{2} M_{2}, \\
& \left\{M_{12}, M_{2}\right\}=8 M_{2}\left(H-2 M_{1}\right)-4\left(1-4 k_{2}^{2}\right) M_{2}+4\left(1-4 k_{1}^{2}\right)\left(H-M_{1}\right), \\
& M_{12}^{2}=16 M_{1} M_{2}\left(H-M_{1}\right)+16 \omega^{2} L_{2}^{2}-4\left(1-4 k_{2}^{2}\right) M_{1}^{2}-4\left(1-4 k_{2}^{2}\right)\left(H-M_{1}\right)^{2} \\
& +4 \omega^{2}\left(1-4 k_{1}^{2}\right)\left(1-4 k_{2}^{2}\right) .
\end{aligned}
$$


We have not included $M_{3}$ in these calculations as it is linearly dependent on $H, M_{1}$ and $M_{2}$ via the relation $M_{3}=M_{2}+\left(e_{2}-e_{1}\right) M_{1}+e_{1} H$. The constants of the motion $M_{1}, M_{2}$ and $H$ generate a quadratic algebra.

(ii) The potential is now

$$
V_{2}(x, y)=\frac{1}{2} \omega^{2}\left(4 x^{2}+y^{2}\right)+\frac{k_{2}^{2}-\frac{1}{4}}{2 y^{2}} .
$$

For $k_{1}=0$ this potential is also known as a Holt potential. ${ }^{20}$ The Schrödinger equation for $k_{1}=0$ has the form

$$
\left(\frac{\partial^{2}}{\partial x^{2}}+\frac{\partial^{2}}{\partial y^{2}}\right) \Psi-\left[\omega^{2}\left(4 x^{2}+y^{2}\right)+\frac{k_{2}^{2}-\frac{1}{4}}{y^{2}}\right] \Psi=-2 E \Psi .
$$

There are two coordinate systems of relevance here: Cartesian coordinates $x, y$ and parabolic coordinates $\xi, \eta$. If we express solutions of the Schrödinger equations in the form

$$
\Psi=e^{-\omega x^{2}-\omega y^{2} / 2} y^{1 / 2 \pm k_{2}} \Phi(x, y),
$$

then the function $\Phi(x, y)$ satisfies the equation $R \Phi=-2 E \Phi$ or

$$
\left[\frac{\partial^{2}}{\partial x^{2}}+\frac{\partial^{2}}{\partial y^{2}}+\frac{\left(1 \pm 2 k_{2}\right)}{y} \frac{\partial}{\partial y}-2 \omega\left(2 x \frac{\partial}{\partial x}+y \frac{\partial}{\partial y}\right)-2 \omega\left(2 \pm k_{2}\right)\right] \Phi(x, y)=-2 E \Phi .
$$

It follows that the operator $R$ maps polynomials (in $x, y^{2}$ ) into polynomials, without increasing the order. Since the original Hamiltonian is self-adjoint with respect to the measure $d x d y$ in the plane, the operator $R$, acting on polynomials in $x, y^{2}$ is self-adjoint with respect to the measure

$$
d \rho(x, y)=e^{-\omega\left(x^{2}+y^{2}\right)} y^{1 \pm 2 k_{2}} d x d y
$$

in the upper half-plane $y>0$. We assume that the positive sign at the $k_{2}$ has to be taken if $k_{2}>$ $\frac{1}{2}$ and both the positive and the negative sign must be taken if $0<k_{2}<\frac{1}{2}$, so that the polynomials have finite norm. ${ }^{16}$

Equation (30) admits a separable solution of the form $\Phi=X(x) Y(y)$ where the function $X(x)$ satisfies the separation equation

$$
\left[\frac{\partial^{2}}{\partial x^{2}}-4 \omega x \frac{\partial}{\partial x}-2 \omega-\lambda\right] X=0 .
$$

If we try solutions of the form $X=\Pi_{m=1}^{q}\left(x-\theta_{m}\right)$, the relations among the $\theta_{m}$ are

$$
4 \omega \theta_{\ell}=\sum_{m \neq \ell} \frac{1}{\theta_{\ell}-\theta_{m}}
$$

and the eigenvalues are $\lambda=-2(2 q+1) \omega$ with solutions $X=H_{q}(\sqrt{2 \omega} x)$. The solutions for the function $Y(y)$ correspond exactly to those given for the potential $V_{1}$ viz

$$
Y(y)=L_{n}^{ \pm k_{2}}\left(y^{2}\right)
$$


The orthonormal eigenfunctions are

$$
\begin{aligned}
\Psi(x, y)= & \sqrt{2} \omega^{(1 / 2)\left(1 \pm k_{2}\right)} \sqrt{\frac{n !}{\Gamma\left(n \pm k_{2}+1\right)}} y^{1 / 2 \pm k_{2}} e^{-1 / 2 \omega y^{2}} L_{n}^{ \pm k_{2}}\left(\omega y^{2}\right) \\
& \times\left(\frac{2 \omega}{\pi}\right)^{1 / 4} \frac{1}{\sqrt{2^{q} q !}} e^{-\omega x^{2}} H_{q}(\sqrt{2 \omega x})
\end{aligned}
$$

The energy $E$ takes the values $E=\omega\left[2(q+n+1) \pm k_{2}\right]$.

In the case of parabolic coordinates we note that

$$
\frac{y^{2}}{\lambda^{2}}+2 x-\lambda^{2}=\frac{\left(\xi^{2}-\lambda^{2}\right)\left(\eta^{2}+\lambda^{2}\right)}{\lambda^{2}} .
$$

If we try a solution of (30) in the form

$$
\Phi(x, y)=\prod_{\ell=1}^{q}\left(\frac{y^{2}}{\lambda_{\ell}^{2}}+2 x-\lambda_{\ell}^{2}\right)
$$

it follows that the $\lambda_{/}$must satisfy

$$
\frac{1}{\lambda_{\ell}^{2}}+\sum_{m \neq \ell} \frac{4}{\lambda_{\ell}^{2}-\lambda_{m}^{2}}-2 \omega \lambda_{\ell}^{2}=0
$$

and $E=\omega\left(2 q+2 \pm k_{2}\right)$. The Schrödinger equation written in terms of parabolic coordinates is

$$
\frac{1}{\xi^{2}+\eta^{2}}\left(\frac{\partial^{2} \Psi}{\partial \xi^{2}}+\frac{\partial^{2} \Psi}{\partial \eta^{2}}\right)+\left[2 E-\omega^{2}\left(\xi^{4}-\xi^{2} \eta^{2}+\eta^{4}\right)+\frac{\frac{1}{4}-k_{2}^{2}}{\xi^{2} \eta^{2}}\right] \Psi=0 .
$$

If we look for separable solutions of the form $\Psi=X_{1}(\xi) X_{2}(\eta)$, the separation equations are

$$
\left[\frac{\partial^{2}}{\partial \mu^{2}}+\left(-\omega^{2} \mu^{6}+\frac{\frac{1}{4}-k_{2}^{2}}{\mu^{2}}+2 E \mu^{2}+\lambda\right)\right] X_{j}=0
$$

where $\mu=\xi$ if $j=1$ and $\mu=\eta$ if $j=2$. The operator whose eigenvalue is $\lambda$ is

$$
\begin{aligned}
\hat{L}_{2} \Psi & =\frac{1}{\xi^{2}+\eta^{2}}\left[\xi^{2} \frac{\partial^{2} \Psi}{\partial \eta^{2}}-\eta^{2} \frac{\partial^{2} \Psi}{\partial \xi^{2}}\right]+\left[\omega^{2} \xi^{2} \eta^{2}+\frac{\frac{1}{4}-k_{2}^{2}}{\xi^{2} \eta^{2}}\right]\left(\xi^{2}-\eta^{2}\right) \Psi \\
& =2 x \frac{\partial^{2} \Psi}{\partial y^{2}}-2 y \frac{\partial^{2} \Psi}{\partial x \partial y}-\frac{\partial \Psi}{\partial x}+\left[2 \omega^{2} x y^{2}+\frac{\frac{1}{4}-k_{2}^{2}}{y^{2}} x\right] \Psi
\end{aligned}
$$

The eigenvalues are

$$
\lambda=2\left(k_{2}+1\right) \prod_{j=1}^{q} \lambda_{j}^{2}\left(\sum_{m=1}^{q} \lambda_{m}^{-2}\right)
$$

If we define the classical operators associated with this separable system as

$$
H=p_{x}^{2}+p_{y}^{2}-\omega^{2}\left(4 x^{2}+y^{2}\right)+\frac{\frac{1}{4}-k_{2}^{2}}{y^{2}}
$$




$$
L_{1}=p_{x}^{2}-4 \omega^{2} x^{2}, \quad L_{2}=2 p_{y}\left(x p_{y}-y p_{x}\right)+2 \omega^{2} x y^{2}+\frac{\frac{1}{4}-k_{2}^{2}}{y^{2}} x
$$

then the defining relations of the quadratic algebra are

$$
\begin{gathered}
L_{12}=\left\{L_{1}, L_{2}\right\}=4\left[p_{x} p_{y}^{2}+\omega^{2} y^{2} p_{x}+2 \frac{\frac{1}{4}-k_{2}^{2}}{y^{2}} p_{x}-4 \omega^{2} x y p_{y}\right], \\
L_{12}^{2}=16\left(H-L_{1}\right)^{2} L_{1}+16 \omega^{2} L_{2}^{2}+16 \omega^{2}\left(1-4 k_{2}^{2}\right), \quad\left\{L_{1}, L_{12}\right\}=-16 \omega^{2} L_{2}, \\
\left\{L_{2}, L_{12}\right\}=16 L_{1}\left(H-L_{1}\right)-8\left(H-L_{1}\right)^{2}-8 \omega^{2}\left(1-4 k_{2}^{2}\right) .
\end{gathered}
$$

(iii) The potential is now

$$
V_{3}=\frac{-\alpha}{\sqrt{x^{2}+y^{2}}}+\frac{1}{4 \sqrt{x^{2}+y^{2}}}\left[\frac{k_{1}^{2}-\frac{1}{4}}{\sqrt{x^{2}+y^{2}}+x}+\frac{k_{2}^{2}-\frac{1}{4}}{\sqrt{x^{2}+y^{2}}-x}\right] .
$$

The Schrödinger equation separates variables in polar coordinates, parabolic coordinates, and modified elliptic coordinates. If we look for solutions of the Schrödinger equation of the form

$$
\Psi=e^{-(1 / 2) \sqrt{-2 E}\left(\xi^{2}+\eta^{2}\right)} \xi^{1 / 2 \pm k_{1}} \eta^{1 / 2 \pm k_{2}} \Phi(\xi, \eta),
$$

where $\xi$ and $\eta$ are parabolic coordinates, we find that the equation for $\Phi(\xi, \eta)$ is $S \Phi=-4 \alpha \Phi$ or

$$
\begin{aligned}
& {\left[\frac{\partial^{2} \Phi}{\partial \xi^{2}}+\frac{\partial^{2} \Phi}{\partial \eta^{2}}+\frac{\left(1 \pm 2 k_{1}\right)}{\xi} \frac{\partial \Phi}{\partial \xi}+\frac{\left(1 \pm 2 k_{2}\right)}{\eta} \frac{\partial \Phi}{\partial \eta}\right.} \\
& \left.\quad+2 \sqrt{-2 E}\left(\xi \frac{\partial \Phi}{\partial \xi}+\eta \frac{\partial \Phi}{\partial \eta}\right)-2 \sqrt{-2 E}\left(2 \pm k_{1} \pm k_{2}\right)\right] \Phi=-4 \alpha \Phi
\end{aligned}
$$

For fixed $E$ we can consider this as an eigenvalue equation for $S$ with eigenvalue $-4 \alpha$. Moreover, $S$ maps polynomials (in $\xi^{2}, \eta^{2}$, or, what is the same thing, in $r=\sqrt{x^{2}+y^{2}}, x$ ) into polynomials, without increasing the order. Since the original Hamiltonian is self-adjoint with respect to the measure $d x d y$ in the plane, the operator $S$, acting on polynomials in $\xi^{2}$ and $\eta^{2}$, is self-adjoint with respect to the measure

$$
d \rho(x, y)=e^{-\sqrt{-2 E}\left(\xi^{2}+\eta^{2}\right)} \xi^{1 \pm 2 k_{1}} \eta^{1 \pm 2 k_{2}}\left(\xi^{2}+\eta^{2}\right) d \xi d \eta .
$$

We assume that the positive sign at the $k_{i}$ has to be taken if $k_{i}>\frac{1}{2}$ and both the positive and the negative sign must be taken if $0<k_{i}<\frac{1}{2}$, so that the polynomials have finite norm. ${ }^{16}$ For polynomial eigenfunctions we must have the quantisation condition

$$
2 \alpha=\sqrt{-2 E}\left[2(q+1) \pm k_{1} \pm k_{2}\right]
$$

for integer $q$.

In polar coordinates with potential $V_{3}$ the Schrödinger equation has the form

$$
\left[\frac{\partial^{2}}{\partial r^{2}}+\frac{1}{r} \frac{\partial}{\partial r}+\frac{1}{r^{2}} \frac{\partial^{2}}{\partial \theta^{2}}\right] \Psi+\left[2 E+\frac{2 \alpha}{r}+\frac{1}{2 r^{2}}\left(\frac{\frac{1}{4}-k_{1}^{2}}{1+\cos \theta}+\frac{\frac{1}{4}-k_{2}^{2}}{1-\cos \theta}\right)\right] \Psi=0 .
$$

If we write solutions $\Psi$ in the form 


$$
\Psi=R(r) S(\theta),
$$

the $\theta$ separation equation is

$$
\frac{d^{2}}{d \theta^{2}} S(\theta)+\frac{1}{16}\left[\frac{1-4 k_{1}^{2}}{\cos ^{2}(\theta / 2)}+\frac{1-4 k_{2}^{2}}{\sin ^{2}(\theta / 2)}\right] S(\theta)=-\lambda^{2} S(\theta),
$$

which has solutions

$$
S(\theta)=\left(\sin \frac{\theta}{2}\right)^{1 / 2 \pm k_{2}}\left(\cos \frac{\theta}{2}\right)^{1 / 2 \pm k_{1}} P_{m}^{\left( \pm k_{1}, \pm k_{2}\right)}(\cos \theta),
$$

where $\lambda=m+\frac{1}{2}\left(1 \pm k_{1} \pm k_{2}\right)$. The separation equation for $R(r)$ is

$$
\left[\frac{\partial^{2}}{\partial r^{2}}+\frac{1}{r} \frac{\partial}{\partial r}-\frac{\lambda^{2}}{r^{2}}+\frac{2 \alpha}{r}+2 E\right] R(r)=0
$$

which has the solution

$$
R_{n \lambda}(r)=e^{-\sqrt{-2 E} r}(2 \sqrt{-2 E} r)^{\lambda} L_{n}^{\lambda}(2 \sqrt{-2 E} r)
$$

where

$$
E=\frac{-2 \alpha^{2}}{\left[2(m+n+1) \pm k_{1} \pm k_{2}\right]^{2}} .
$$

The orthonormal basis of eigenfunctions is

$$
\Psi=\sqrt{\frac{\alpha n !}{\Gamma(2 n+2 \lambda+1)(2 n+2 \lambda+1)}} R_{n \lambda}(r) \Phi_{n}^{\left( \pm k_{2}, \pm k_{1}\right)}\left(\frac{\theta}{2}\right) .
$$

The operator whose eigenvalue is $\mu^{2}$ is, in Cartesian coordinates,

$$
L_{1} \Psi=\left[\left(x \frac{\partial}{\partial y}-y \frac{\partial}{\partial x}\right)^{2}-\frac{1}{2} \sqrt{x^{2}+y^{2}}\left(\frac{k_{1}^{2}-\frac{1}{4}}{\sqrt{x^{2}+y^{2}}+x}+\frac{k_{2}^{2}-\frac{1}{4}}{\sqrt{x^{2}+y^{2}}-x}\right)\right] \Psi
$$

In parabolic coordinates Schrödinger's equation has the form

$$
H \Psi=\frac{1}{\xi^{2}+\eta^{2}}\left[\frac{\partial^{2} \Psi}{\partial \xi^{2}}+\frac{\partial^{2} \Psi}{\partial \eta^{2}}\right]+\left[4 \alpha+\left(\frac{\frac{1}{4}-k_{1}^{2}}{\xi^{2}}+\frac{\frac{1}{4}-k_{2}^{2}}{\eta^{2}}\right)\right] \Psi=-2 E \Psi .
$$

The separation equations are

$$
\frac{\partial^{2}}{\partial \mu^{2}} X_{j}+\left(\frac{\frac{1}{4}-k_{j}^{2}}{\mu^{2}}+2 E \mu^{2}+\lambda_{j}\right) X_{j}=0
$$


with $\lambda_{1}+\lambda_{2}=4 \alpha$. The orthonormal bound state eigenfunctions of Schrödinger's equation in these coordinates are

$$
\begin{aligned}
\Psi= & X_{1}(\xi) X_{2}(\eta) \\
= & \sqrt{\frac{\alpha}{\left[2\left(n_{1}+n_{2}\right) \pm k_{1} \pm k_{2}+2\right]^{3}}} \sqrt{\frac{n_{1} ! n_{2} !}{\Gamma\left(n_{1} \pm k_{1}+1\right) \Gamma\left(n_{2} \pm k_{2}+1\right)}} e^{-(1 / 2) \sqrt{-2 E}\left(\xi^{2}+\eta^{2}\right)} \\
& \times(\sqrt{-2 E} \xi)^{1 / 2 \pm k_{1}}(\sqrt{-2 E} \eta)^{1 / 2 \pm k_{2}} L_{n_{1}}^{ \pm k_{1}}\left(\sqrt{-2 E} \xi^{2}\right) L_{n_{2}}^{ \pm k_{2}}\left(\sqrt{-2 E} \eta^{2}\right),
\end{aligned}
$$

where $\lambda_{j}=2 \sqrt{-2 E}\left(2 n_{j} \pm k_{j}+1\right), j=1,2$, and

$$
E=-\frac{2 \alpha^{2}}{\left[2\left(n_{1}+n_{2}\right) \pm k_{1} \pm k_{2}+2\right]^{2}} .
$$

If we choose $\lambda_{1}=2 \alpha+\lambda$ and $\lambda_{2}=2 \alpha-\lambda$, then the symmetry operator with eigenvalue $\lambda$ is

$$
\begin{aligned}
L_{2} \Psi= & 2 x \frac{\partial^{2} \Psi}{\partial y^{2}}-2 y \frac{\partial^{2} \Psi}{\partial x \partial y}-\frac{\partial \Psi}{\partial x}+\left[-\left(k_{1}^{2}-\frac{1}{4}\right) \frac{\sqrt{x^{2}+y^{2}}-x}{\sqrt{x^{2}+y^{2}}\left(\sqrt{x^{2}+y^{2}}+x\right)}\right. \\
& \left.+\left(k_{2}^{2}-\frac{1}{4}\right) \frac{\sqrt{x^{2}+y^{2}}+x}{\sqrt{x^{2}+y^{2}}\left(\sqrt{x^{2}+y^{2}}-x\right)}+\frac{4 \alpha}{\sqrt{x^{2}+y^{2}}}\right] \Psi \\
= & \frac{1}{\xi^{2}+\eta^{2}}\left[\xi^{2} \frac{\partial^{2} \Psi}{\partial \eta^{2}}-\eta^{2} \frac{\partial^{2} \Psi}{\partial \xi^{2}}\right]+\left[\left(\frac{1}{4}-k_{1}^{2}\right) \frac{\eta^{2}}{\xi^{2}\left(\xi^{2}+\eta^{2}\right)}\right. \\
& \left.+\left(k_{2}^{2}-\frac{1}{4}\right) \frac{\xi^{2}}{\eta^{2}\left(\xi^{2}+\eta^{2}\right)}+2 \alpha \frac{\xi^{2}-\eta^{2}}{\xi^{2}+\eta^{2}}\right] \Psi .
\end{aligned}
$$

For the case of elliptical coordinates we proceed as follows. The normal elliptical coordinates will not separate variables. However, if we compare (12) and (39) we are motivated to choose coordinates such that

$$
\xi^{2}=\frac{\left(u_{1}-e_{1}\right)\left(u_{2}-e_{1}\right)}{e_{1}-e_{2}}, \quad \eta^{2}=\frac{\left(u_{1}-e_{2}\right)\left(u_{2}-e_{2}\right)}{e_{2}-e_{1}} .
$$

If we then try a solution of (39) in the form

$$
\Phi(\xi, \eta)=\prod_{m=1}^{q}\left[\frac{\xi^{2}}{\theta_{m}-e_{1}}+\frac{\eta^{2}}{\theta_{m}-e_{2}}-1\right]
$$

we see that the $\theta_{m}$ must satisfy

$$
\frac{1 \pm k_{1}}{\theta_{m}-e_{1}}+\frac{1 \pm k_{2}}{\theta_{m}-e_{2}}+\sum_{j \neq m} \frac{2}{\theta_{m}-\theta_{j}}-\sqrt{-2 E}=0
$$

and the quantization condition (40). In this choice of coordinates the Schrödinger equation has the form 


$$
\begin{aligned}
& \frac{4\left(u_{1}-e_{1}\right)\left(u_{1}-e_{2}\right)}{u_{1}-u_{2}}\left[\frac{\partial^{2} \Phi}{\partial u_{1}^{2}}+\frac{1}{2}\left(\frac{1}{u_{1}-e_{1}}+\frac{1}{u_{1}-e_{2}}\right) \frac{\partial \Phi}{\partial u_{1}}\right] \\
& +\frac{4\left(u_{2}-e_{1}\right)\left(u_{2}-e_{2}\right)}{u_{2}-u_{1}}\left[\frac{\partial^{2} \Phi}{\partial u_{2}^{2}}+\frac{1}{2}\left(\frac{1}{u_{2}-e_{1}}+\frac{1}{u_{2}-e_{2}}\right) \frac{\partial \Phi}{\partial u_{2}}\right] \\
& +\left[2 E\left(u_{1}+u_{2}-e_{1}-e_{2}\right)+\frac{\left(\frac{1}{4}-k_{1}^{2}\right)\left(e_{2}-e_{1}\right)}{\left(u_{1}-e_{1}\right)\left(u_{2}-e_{1}\right)}+\frac{\left(\frac{1}{4}-k_{2}^{2}\right)\left(e_{1}-e_{2}\right)}{\left(u_{1}-e_{2}\right)\left(u_{2}-e_{2}\right)}+4 \alpha\right] \Phi=0 .
\end{aligned}
$$

This equation admits separable solutions $\Phi=U_{1}\left(u_{1}\right) U_{2}\left(u_{2}\right)$ and separation equations

$$
\begin{gathered}
\left\{4\left(u_{i}-e_{1}\right)\left(u_{i}-e_{2}\right)\left(\frac{\partial^{2}}{\partial u_{i}^{2}}+\frac{1}{2}\left[\frac{1}{u_{i}-e_{1}}+\frac{1}{u_{i}-e_{2}}\right] \frac{\partial}{\partial u_{i}}\right)+2 E\left[u_{i}^{2}-\left(e_{1}+e_{2}\right) u_{i}\right]\right. \\
\left.+\frac{\left(k_{1}^{2}-\frac{1}{4}\right)\left(e_{1}-e_{2}\right)}{u_{1}-e_{1}}+\frac{\left(k_{2}^{2}-\frac{1}{4}\right)\left(e_{2}-e_{1}\right)}{u_{1}-e_{2}}+4 \alpha u_{i}+\lambda\right\} U_{i}\left(u_{i}\right)=0, \quad i=1,2 .
\end{gathered}
$$

The additional operator describing this coordinate system is

$$
\begin{aligned}
\hat{L}_{3} \Phi= & \frac{4}{\left(u_{1}-u_{2}\right)\left(u_{1}+u_{2}-e_{1}-e_{2}\right)}\left\{u_{2}\left(u_{1}-e_{1}\right)\left(u_{1}-e_{2}\right)\left(\frac{\partial^{2} \Phi}{\partial u_{1}^{2}}+\frac{1}{2}\left[\frac{1}{u_{1}-e_{1}}+\frac{1}{u_{1}-e_{2}}\right] \frac{\partial \Phi}{\partial u_{1}}\right)\right. \\
& \left.-u_{1}\left(u_{2}-e_{1}\right)\left(u_{2}-e_{2}\right)\left(\frac{\partial^{2} \Phi}{\partial u_{2}^{2}}+\frac{1}{2}\left[\frac{1}{u_{2}-e_{1}}+\frac{1}{u_{2}-e_{2}}\right] \frac{\partial \Phi}{\partial u_{2}}\right)\right\} \\
& +\frac{k_{1}^{2}-\frac{1}{4}}{\left(u_{1}-e_{1}\right)\left(u_{2}-e_{1}\right)}\left\{u_{1}+u_{2}-e_{1}-e_{2}-\frac{\left[\left(u_{1}-e_{2}\right)\left(u_{2}-e_{2}\right)+e_{1} e_{2}\right]}{\left(u_{1}+u_{2}-e_{1}-e_{2}\right)}\right\} \Phi \\
& +\frac{k_{2}^{2}-\frac{1}{4}}{\left(u_{1}-e_{2}\right)\left(u_{2}-e_{2}\right)}\left\{u_{1}+u_{2}-e_{1}-e_{2}-\frac{\left[\left(u_{1}-e_{1}\right)\left(u_{2}-e_{1}\right)+e_{1} e_{2}\right]}{\left(u_{1}+u_{2}-e_{1}-e_{2}\right)}\right\} \Phi \\
& +\frac{4 \alpha u_{1} u_{2}}{u_{1}+u_{2}-e_{1}-e_{2}} \Phi
\end{aligned}
$$

In Cartesian coordinates the solutions have the form

$$
\begin{aligned}
\Psi= & e^{-\sqrt{-2 E}\left(x^{2}+y^{2}\right)}\left(\sqrt{x^{2}+y^{2}}+x\right)^{(1 / 4)\left(1 \pm 2 k_{1}\right)}\left(\sqrt{x^{2}+y^{2}}-x\right)^{(1 / 4)\left(1 \pm 2 k_{2}\right)} \\
& \times \prod_{m=1}^{q}\left(\frac{\sqrt{x^{2}+y^{2}}+x}{\theta_{m}-e_{1}}+\frac{\sqrt{x^{2}+y^{2}}-x}{\theta_{m}-e_{2}}-1\right) .
\end{aligned}
$$

Acting on the functions $\Psi$ the operator $L_{3}$ has the form 


$$
\begin{aligned}
L_{3} \Psi= & {\left[\left(\xi \frac{\partial}{\partial \eta}-\eta \frac{\partial}{\partial \xi}\right)^{2}+e_{2} \frac{\partial^{2}}{\partial \xi^{2}}+e_{1} \frac{\partial^{2}}{\partial \eta^{2}}-\left(e_{2} \xi^{2}+e_{1} \eta^{2}+e_{1} e_{2}\right) H\right.} \\
& \left.+\left(\frac{1}{4}-k_{1}^{2}\right) \frac{\left(\xi^{2}+\eta^{2}+e_{2}\right)}{\xi^{2}}+\left(\frac{1}{4}-k_{2}^{2}\right) \frac{\left(\xi^{2}+\eta^{2}+e_{2}\right)}{\eta^{2}}\right] \Psi \\
= & -4\left(x \frac{\partial}{\partial y}-y \frac{\partial}{\partial x}\right)^{2}-\left(e_{1}-e_{2}\right)\left(2 x \frac{\partial^{2}}{\partial y^{2}}-2 y \frac{\partial^{2}}{\partial x \partial y}-\frac{\partial}{\partial x}\right)-e_{1} e_{2} H \\
& -\frac{2 \alpha}{\sqrt{x^{2}+y^{2}}}\left[e_{1}\left(\sqrt{x^{2}+y^{2}}-x\right)+e_{2}\left(\sqrt{x^{2}+y^{2}}+x\right)\right]-\left(\frac{1}{4}-k_{1}^{2}\right) \\
& \times \frac{\left(e_{1}-e_{2}\right)\left(\sqrt{x^{2}+y^{2}}-x\right)}{2 \sqrt{x^{2}+y^{2}}\left(\sqrt{x^{2}+y^{2}}+x\right)}-\left(\frac{1}{4}-k_{2}^{2}\right) \frac{\left(e_{1}-e_{2}\right)\left(\sqrt{x^{2}+y^{2}}+x\right)}{2 \sqrt{x^{2}+y^{2}}\left(\sqrt{x^{2}+y^{2}}-x\right)} \\
& \left.+\left(\frac{1}{4}-k_{1}^{2}\right) \frac{\sqrt{x^{2}+y^{2}}-x}{\sqrt{x^{2}+y^{2}}+x}+\left(\frac{1}{4}-k_{2}^{2}\right) \frac{\sqrt{x^{2}+y^{2}}+x}{\sqrt{x^{2}+y^{2}}-x}+\frac{1}{2}-k_{1}^{2}-k_{2}^{2}\right] \Psi,
\end{aligned}
$$

where in Cartesian coordinates

$$
H \Psi=\frac{\partial^{2} \Psi}{\partial x^{2}}+\frac{\partial^{2} \Psi}{\partial y^{2}}+\left[\frac{-\alpha}{\sqrt{x^{2}+y^{2}}}+\frac{1}{4 \sqrt{x^{2}+y^{2}}}\left[\frac{k_{1}^{2}-\frac{1}{4}}{\sqrt{x^{2}+y^{2}}+x}+\frac{k_{2}^{2}-\frac{1}{4}}{\sqrt{x^{2}+y^{2}}-x}\right]\right] \Psi .
$$

The eigenvalues of the operator $L_{3}$ are

$$
\begin{aligned}
\lambda= & -\left(1 \pm k_{1} \pm k_{2}\right)^{2}-2 \sqrt{-2 E}\left[e_{2}\left(1 \pm k_{1}\right)-2 e_{1}\left(1 \pm k_{2}\right)\right] \\
& +2 E e_{1} e_{2}-4 \sum_{m=1}^{q}\left[e_{2} \frac{k_{1}+1}{\theta_{m}-e_{1}}+e_{1} \frac{k_{2}+1}{\theta_{m}-e_{2}}\right] .
\end{aligned}
$$

The corresponding quadratic algebra can be generated by the classical constants

$$
\begin{gathered}
L_{1}=\left(\xi p_{\eta}-\eta p_{\xi}\right)^{2}+\left(\xi^{2}+\eta^{2}\right)\left[\frac{\frac{1}{4}-k_{1}^{2}}{\xi^{2}}+\frac{\frac{1}{4}-k_{2}^{2}}{\eta^{2}}\right], \\
L_{2}=\frac{1}{\xi^{2}+\eta^{2}}\left[\xi^{2} p_{\eta}^{2}-\eta^{2} p_{\xi}^{2}-\frac{\eta^{2}}{\xi^{2}}\left(\frac{1}{4}-k_{1}^{2}\right)-\frac{\xi^{2}}{\eta^{2}}\left(\frac{1}{4}-k_{2}^{2}\right)+2 \alpha\left(\xi^{2}-\eta^{2}\right)\right], \\
L_{3}=\left(\xi p_{\eta}-\eta p_{\xi}\right)^{2}+e_{2} p_{\xi}^{2}+e_{1} p_{\eta}^{2}-\left(e_{2} \xi^{2}+e_{1} \eta^{2}\right) H \\
+\left(\frac{1}{4}-k_{1}^{2}\right) \frac{\left(\xi^{2}+\eta^{2}+e_{2}\right)}{\xi^{2}}+\left(\frac{1}{4}-k_{2}^{2}\right) \frac{\left(\xi^{2}+\eta^{2}+e_{2}\right)}{\eta^{2}}, \\
H=\frac{1}{\xi^{2}+\eta^{2}}\left[p_{\xi}^{2}+p_{\eta}^{2}+4 \alpha+\left(\frac{\frac{1}{4}-k_{1}^{2}}{\xi^{2}}+\frac{\frac{1}{4}-k_{2}^{2}}{\eta^{2}}\right)\right] .
\end{gathered}
$$

Here, $L_{1}, L_{2}$, and $L_{3}$ are functionally dependent via the relation 


$$
\begin{aligned}
L_{3}^{2}= & L_{1}^{2}+2\left(e_{1}-e_{2}\right) L_{1} L_{2}+\left(e_{1}-e_{2}\right)^{2} L_{2}^{2}-2\left[\left(k_{1}+k_{2}\right)+2\left(e_{1}+e_{2}\right) \alpha\right] L_{1} \\
& -\left[4 \alpha^{2}\left(e_{1}^{2}-e_{2}^{2}\right)+2\left(e_{1}-e_{2}\right)\left(k_{1}+k_{2}\right)\right] L_{2}-\left(k_{1}+k_{2}+2 \alpha\left(e_{1}+e_{2}\right)\right)^{2} .
\end{aligned}
$$

The only additional quantity that we need is

$$
\begin{aligned}
L_{12}= & \left\{L_{1}, L_{2}\right\}=\frac{1}{\xi^{2}+\eta^{2}}\left[4\left(\xi p_{\eta}-\eta p_{\xi}\right)^{2}\left(\xi p_{\xi}-\eta p_{\eta}\right)-16 \xi \eta\left(\xi p_{\eta}-\eta p_{\xi}\right)\right. \\
& +\left[\left(4 \frac{\xi^{3}}{\eta^{2}}+8 \xi\right)\left(\frac{1}{4}-k_{2}^{2}\right)+4 \frac{\eta^{2}}{\xi}\left(\frac{1}{4}-k_{1}^{2}\right)\right] p_{\xi} \\
& \left.-\left[\left(4 \frac{\eta^{3}}{\xi^{2}}+8 \eta\right)\left(\frac{1}{4}-k_{1}^{2}\right)+4 \frac{\xi^{2}}{\eta}\left(\frac{1}{4}-k_{2}^{2}\right)\right] p_{\eta}\right] .
\end{aligned}
$$

The nontrivial independent commutation relations are

$$
\left\{L_{12}, L_{1}\right\}=-16 L_{1} L_{2}+32 \alpha\left(k_{1}^{2}-k_{2}^{2}\right), \quad\left\{L_{12}, L_{2}\right\}=-16 H L_{1}+8 L_{2}^{2} .
$$

We should also observe the relations

$$
\begin{gathered}
\left\{L_{1}, L_{3}\right\}=\left(e_{1}-e_{2}\right)\left\{L_{1}, L_{2}\right\}, \quad\left\{L_{1}, L_{2}\right\}=\left\{L_{3}, L_{2}\right\}, \\
\left\{L_{12}, L_{3}\right\}=16\left(e_{1}-e_{2}\right) H L_{1}-8\left(e_{1}-e_{2}\right) L_{2}^{2}-16 L_{1} L_{2} \\
-16\left(e_{1}-e_{2}\right) H-32 \alpha\left(\alpha\left(e_{1}-e_{2}\right)+\left(k_{1}^{2}-k_{2}^{2}\right)\right) .
\end{gathered}
$$

(iv) The fourth potential is

$$
V_{4}=\frac{-\alpha}{\sqrt{x^{2}+y^{2}}}+\frac{B_{1}}{4} \frac{\sqrt{\sqrt{x^{2}+y^{2}}+x}}{\sqrt{x^{2}+y^{2}}}+\frac{B_{2}}{4} \frac{\sqrt{\sqrt{x^{2}+y^{2}}-x}}{\sqrt{x^{2}+y^{2}}} .
$$

The corresponding Schrödinger equation separates in two versions of parabolic coordinates.

In regular parabolic coordinates (2) the Schrödinger equation has the form

$$
\frac{1}{\xi^{2}+\eta^{2}}\left[\frac{\partial^{2} \Psi}{\partial \xi^{2}}+\frac{\partial^{2} \Psi}{\partial \eta^{2}}+\left(4 \alpha-B_{1} \xi-B_{2} \eta\right) \Psi\right]=-2 E \Psi .
$$

If we write the solutions $\Psi$ in the form $X(\xi) Y(\eta)$, the separation equations are

$$
\frac{\partial^{2} X}{\partial \xi^{2}}+\left(2 \alpha-\lambda-B_{1} \xi+2 E \xi^{2}\right) X=0, \quad \frac{\partial^{2} Y}{\partial \eta^{2}}+\left(2 \alpha+\lambda-B_{2} \eta+2 E \eta^{2}\right) Y=0 .
$$

The solutions of these equations are

$$
X(\xi)=e^{(1 / 2 \sqrt{-2 E})\left[-2 E \xi^{2}+B_{1} \xi\right]} H_{n}\left(\sqrt{-2 E}\left(\xi-\frac{B_{1}}{4 E}\right)\right)
$$

and

$$
Y(\eta)=e^{(1 / 2 \sqrt{-2 E})\left[-2 E \eta^{2}+B_{2} \eta\right]} H_{m}\left(\sqrt{-2 E}\left(\eta-\frac{B_{2}}{4 E}\right)\right)
$$

where 


$$
2 \alpha-\lambda-\frac{B_{1}^{2}}{8 E}=-(n+1) \sqrt{-2 E}, \quad 2 \alpha+\lambda-\frac{B_{2}^{2}}{8 E}=-(m+1) \sqrt{-2 E} .
$$

From this we see that the energy eigenstates are solutions of

$$
4 \alpha-\frac{1}{8 E}\left(B_{1}^{2}+B_{2}^{2}\right)=-(m+n+2) \sqrt{-2 E} .
$$

Moreover, it follows from (59) and (60) that

$$
\Psi(\xi, \eta)=e^{(1 / 2 \sqrt{-2 E})\left[-2 E\left(\xi^{2}+\eta^{2}\right)+B_{1} \xi+B_{2} \eta\right]} \Phi(\xi, \eta),
$$

where $\Phi$ is a polynomial in $\xi$ and $\eta$. If (58) is written as a differential equation for $\Phi$, it can be interpreted as an eigenvalue equation, with eigenvalue $-4 \alpha$, for an $E$-dependent operator acting on a space of polynomials. The operator whose eigenvalue is $\lambda$ is

$$
\begin{aligned}
L_{1} \Psi= & \frac{1}{\xi^{2}+\eta^{2}}\left\{\eta^{2} \frac{\partial^{2} \Psi}{\partial \xi^{2}}-\xi^{2} \frac{\partial^{2} \Psi}{\partial \eta^{2}}+\left[-B_{1} \xi \eta^{2}+B_{2} \eta \xi^{2}+2\left(\eta^{2}-\xi^{2}\right) \alpha\right] \Psi\right\} \\
= & -2 x \frac{\partial^{2} \Psi}{\partial y^{2}}+2 y \frac{\partial^{2} \Psi}{\partial x \partial y}+\frac{\partial \Psi}{\partial x}+\left[\frac{B_{1}}{2} \sqrt{\sqrt{x^{2}+y^{2}}+x}\left(1-\frac{x}{\sqrt{x^{2}+y^{2}}}\right)\right. \\
& \left.+\frac{B_{2}}{2} \sqrt{\sqrt{x^{2}+y^{2}}-x}\left(1+\frac{x}{\sqrt{x^{2}+y^{2}}}\right)-\frac{2 x \alpha}{\sqrt{x^{2}+y^{2}}}\right] \Psi
\end{aligned}
$$

The second coordinate system is a variant of parabolic coordinates (3) in terms of which the Schrödinger equation has the form

$$
\frac{1}{\mu^{2}+\nu^{2}}\left\{\frac{\partial^{2} \Psi}{\partial \mu^{2}}+\frac{\partial^{2} \Psi}{\partial \nu^{2}}+\left[4 \alpha-\frac{B_{1}}{\sqrt{2}}(\mu+\nu)-\frac{B_{2}}{\sqrt{2}}(\mu-\nu)\right] \Psi\right\}=-2 E \Psi
$$

The separation equations for $\Psi=X(\mu) Y(\nu)$ are

$$
\begin{aligned}
& \frac{\partial^{2} X}{\partial \mu^{2}}+\left(2 \alpha-\lambda-\frac{1}{\sqrt{2}}\left(B_{1}+B_{2}\right) \mu+2 E \mu^{2}\right) X=0, \\
& \frac{\partial^{2} Y}{\partial \nu^{2}}+\left(2 \alpha+\lambda-\frac{1}{\sqrt{2}}\left(B_{1}-B_{2}\right) \nu+2 E \nu^{2}\right) Y=0 .
\end{aligned}
$$

The operator whose eigenvalue is $\lambda$ is

$$
\begin{aligned}
L_{2} \Psi= & \frac{1}{\mu^{2}+\nu^{2}}\left[\nu^{2} \frac{\partial^{2} \Psi}{\partial \mu^{2}}-\mu^{2} \frac{\partial^{2} \Psi}{\partial \nu^{2}}-\frac{\mu \nu^{2}}{\sqrt{2}}\left(B_{1}+B_{2}\right) \Psi+\frac{\nu \mu^{2}}{\sqrt{2}}\left(B_{1}-B_{2}\right) \Psi+2\left(\nu^{2}-\mu^{2}\right) \alpha \Psi\right] \\
= & -2 y \frac{\partial^{2} \Psi}{\partial x^{2}}+2 x \frac{\partial^{2} \Psi}{\partial x \partial y}+\frac{\partial \Psi}{\partial y}+\left[-\frac{1}{2 \sqrt{2}}\left(B_{1}+B_{2}\right) \sqrt{\sqrt{x^{2}+y^{2}}+x}\left(1-\frac{x}{\sqrt{x^{2}+y^{2}}}\right)\right. \\
& \left.+\frac{1}{2 \sqrt{2}}\left(B_{1}-B_{2}\right) \sqrt{\sqrt{x^{2}+y^{2}}-x}\left(1+\frac{x}{\sqrt{x^{2}+y^{2}}}\right)-\frac{2 x \alpha}{\sqrt{x^{2}+y^{2}}}\right] \Psi
\end{aligned}
$$


The space of polynomials in $\xi, \eta$ coincides with the space of polynomials in $\nu, \mu$. The classical operators associated with this separable system are, using the $\xi, \eta$ coordinates,

$$
\begin{gathered}
L_{1}=\frac{1}{\xi^{2}+\eta^{2}}\left[\eta^{2} p_{\xi}^{2}-\xi^{2} p_{\eta}^{2}-B_{1} \xi \eta^{2}+B_{2} \eta \xi^{2}+2\left(\eta^{2}-\xi^{2}\right) \alpha\right], \\
L_{2}=\frac{1}{\xi^{2}+\eta^{2}}\left[\left(\xi p_{\xi}-\eta p_{\eta}\right)\left(\xi p_{\eta}-\eta p_{\xi}\right)-4 \xi \eta \alpha+\frac{1}{2}\left(\xi^{2}-\eta^{2}\right)\left(\eta B_{1}-\xi B_{2}\right)\right], \\
H=\frac{1}{\xi^{2}+\eta^{2}}\left[p_{\xi}^{2}+p_{\eta}^{2}+\left(4 \alpha-B_{1} \xi-B_{2} \eta\right)\right],
\end{gathered}
$$

together with

$$
\begin{aligned}
L_{12}= & \left\{L_{1}, L_{2}\right\}=\frac{1}{\xi^{2}+\eta^{2}}\left[2\left(\xi p_{\eta}-\eta p_{\xi}\right)\left(p_{\xi}^{2}+p_{\eta}^{2}\right)+8 \alpha\left(\xi p_{\eta}-\eta p_{\xi}\right)\right. \\
& \left.+\left(\left(\eta^{2}-\xi^{2}\right) p_{\eta}+2 \xi \eta p_{\xi}\right) B_{1}+\left(\left(\eta^{2}-\xi^{2}\right) p_{\xi}+2 \xi \eta p_{\eta}\right) B_{2}\right] .
\end{aligned}
$$

The defining relations of the quadratic algebra are

$$
\begin{gathered}
L_{12}^{2}=4 L_{1}^{2} H+4 L_{2}^{2} H-16 \alpha^{2} H+\left(B_{2}^{2}-B_{1}^{2}\right) L_{1}-2 B_{1} B_{2} L_{2}-2 \alpha^{2}\left(B_{1}^{2}+B_{2}^{2}\right), \\
\left\{L_{12}, L_{1}\right\}=-4 L_{1}^{2} H+B_{1} B_{2}, \quad\left\{L_{12}, L_{2}\right\}=4 L_{1} H-\frac{1}{2}\left(B_{1}^{2}-B_{2}^{2}\right) .
\end{gathered}
$$

There are, of course, quantum analogs for all the quadratic algebras that occur for each potential. These operator algebras can be obtained via the replacement of the Poisson bracket by the commutator bracket. [Indeed the relations (64) for the classical version of the algebras remain unchanged under this replacement.] One can ask the question what are the consequences of such an algebra on the eigenfunction spaces of the operators $L_{1}$ and $L_{2}$ ? Let the eigenspace corresponding to a fixed bound state energy $E$ be of dimension $N$. This eigenspace can be spanned by eigenfunctions of both $L_{1}$ and $L_{2}$. Let the corresponding eigenvectors be $\phi_{m}$ and $\psi_{m}$, respectively, satisfying

$$
L_{1} \phi_{m}=\lambda_{m} \phi_{m}, \quad L_{2} \psi_{n}=\rho_{n} \psi_{n},
$$

where $\lambda_{n}=2 \alpha-B_{1}^{2} / 8 E-(2 n+1) \sqrt{-2 E}$ and $\rho_{m}=2 \alpha-\left(B_{1}+B_{2}\right)^{2} / 16 E-(2 m+1) \sqrt{-2 E}$. Using the quantum version of the defining relations of the quadratic algebra, what can we deduce? If we use the second of the relations (64) acting on $\psi_{n}$ and write $L_{1} \psi_{n}=\sum_{\tau=1}^{N} C_{n \tau} \psi_{\tau}$ we deduce that

$$
\left[\left(\rho_{n}-\rho_{\tau}\right)^{2}+8 E\right] C_{n \tau}=-\left[\frac{1}{2}\left(B_{1}^{2}-B_{2}^{2}\right)+16 \alpha E\right] \delta_{n \tau} .
$$

This relation implies that $C_{n n}=-\left[\frac{1}{2}\left(B_{1}^{2}-B_{2}^{2}\right)+16 \alpha E\right] / 8 E$ and $C_{p q} \neq 0$ if $|p-q| \leqslant 1$ and zero otherwise. Here we have used the relation $\left(\rho_{n}-\rho_{\tau}\right)^{2}+8 E=0$ for $|n-\tau|=1$. If we apply the second relation to the function $\psi_{n}$ we obtain the relation

$$
\sum_{\tau=1}^{N} C_{n \tau} C_{\tau \sigma}\left(2 \rho_{\tau}-\rho_{n}-\rho_{\sigma}\right)=\left(8 E \rho_{n}+B_{1} B_{2}+16 \alpha E\right) \delta_{n \sigma} .
$$

If we assume that the basis functions $\psi_{n}$ are orthonormal and observe that the operator $L_{1}$ is self-adjoint, then we can assume $C_{n+1, n}=C_{n, n+1}^{*}$. The only nontrivial consequence of this relation is when $n=\sigma$. The result is 


$$
4 \sqrt{-2 E}\left(\left|C_{n, n+1}\right|^{2}-\left|C_{n-1, n}\right|^{2}\right)=8 E \rho_{n}+B_{1} B_{2}+16 \alpha E .
$$

If we act on the functions $\phi_{m}$, we generate similar relations. In fact, if we write $L_{2} \phi_{m}=\sum_{\tau=1}^{N} D_{m \tau} \phi_{\tau}$, the corresponding results are

$$
D_{n n}=-\left(B_{1} B_{2}+16 \alpha E\right) / 8 E
$$

and $D_{p q} \neq 0$ if $|p-q| \leqslant 1$ and is zero otherwise. Furthermore,

$$
4 \sqrt{-2 E}\left(\left|D_{n, n+1}\right|^{2}-\left|D_{n-1, n}\right|^{2}\right)=-8 \lambda_{\sigma} E-\frac{1}{2}\left(B_{1}^{2}-B_{2}^{2}\right)-16 \alpha E .
$$

From these relations we can, in principle, calculate the matrices $C_{p q}$ and $D_{p q}$. Once these are known, relations are also implied for the matrices relating the two bases. If we denote $\left\langle\phi_{m}, \psi_{q}\right\rangle=A_{m q}$, then these matrices must satisfy

$$
C_{t r}=\sum_{m=1}^{N} \lambda_{m} A_{m r}^{*} A_{m t}, \quad D_{t r}=\sum_{m=1}^{N} \rho_{m} A_{m r} A_{m t}^{*}
$$

Thus, from the consequences of the quadratic algebra we can, in principle, calculate the overlap functions $A_{r s}$. The only ambiguity is in the choice of the phases of the wavefunctions.

\section{THE TWO-DIMENSIONAL SPHERE}

In the case of the two-dimensional sphere there are two potentials that are separable in more than one coordinate system, and these each separate in two systems. To work with the twodimensional sphere it is convenient to use projective coordinates: $s_{i}, i=1,2,3, s_{1}^{2}+s_{2}^{2}+s_{3}^{2}=1$.

(i) The first superintegrable potential on the sphere is

$$
V_{1}=\frac{1}{2}\left(\frac{k_{1}^{2}-\frac{1}{4}}{s_{1}^{2}}+\frac{k_{2}^{2}-\frac{1}{4}}{s_{2}^{2}}+\frac{k_{3}^{2}-\frac{1}{4}}{s_{3}^{2}}\right) .
$$

In terms of spherical coordinates

$$
s_{1}=\sin \theta \cos \varphi, \quad s_{2}=\sin \theta \sin \varphi, \quad s_{3}=\cos \theta,
$$

the corresponding Schrödinger equation has the form

$$
\begin{gathered}
{\left[\left(s_{1} \frac{\partial}{\partial s_{2}}-s_{2} \frac{\partial}{\partial s_{1}}\right)^{2}+\left(s_{1} \frac{\partial}{\partial s_{3}}-s_{3} \frac{\partial}{\partial s_{1}}\right)^{2}+\left(s_{3} \frac{\partial}{\partial s_{2}}-s_{2} \frac{\partial}{\partial s_{3}}\right)^{2}\right] \Psi} \\
-\left[\frac{k_{1}^{2}-\frac{1}{4}}{s_{1}^{2}}+\frac{k_{2}^{2}-\frac{1}{4}}{s_{2}^{2}}+\frac{k_{3}^{2}-\frac{1}{4}}{s_{3}^{2}}\right] \Psi=-2 E \Psi,
\end{gathered}
$$

or, in terms of $\theta$ and $\varphi$,

$$
\frac{1}{\sin \theta} \frac{\partial}{\partial \theta}\left(\sin \theta \frac{\partial \Psi}{\partial \theta}\right)+\frac{1}{\sin ^{2} \theta} \frac{\partial^{2} \Psi}{\partial \varphi^{2}}+\frac{1}{\sin ^{2} \theta}\left(\frac{\frac{1}{4}-k_{1}^{2}}{\cos ^{2} \varphi}+\frac{\frac{1}{4}-k_{2}^{2}}{\sin ^{2} \varphi}\right) \Psi+\frac{\frac{1}{4}-k_{3}^{2}}{\cos ^{2} \theta} \Psi=-2 E \Psi .
$$

This equation admits separable solutions of the form $\Psi=T(\theta) \Phi(\varphi)$ such that 


$$
\begin{gathered}
\frac{\partial^{2} \Phi}{\partial \varphi^{2}}+\left(\frac{\frac{1}{4}-k_{1}^{2}}{\cos ^{2} \varphi}+\frac{\frac{1}{4}-k_{2}^{2}}{\sin ^{2} \varphi}\right) \Phi=-\lambda \Phi, \\
\frac{1}{\sin \theta} \frac{\partial}{\partial \theta}\left(\sin \theta \frac{\partial T}{\partial \theta}\right)+\frac{1}{\sin ^{2} \theta} \lambda T+\frac{\frac{1}{4}-k_{3}^{2}}{\cos ^{2} \theta} T=-2 E T .
\end{gathered}
$$

These equations have the solutions

$$
\Phi=(\sin \varphi)^{k_{2}+1 / 2}(\cos \varphi)^{k_{1}+1 / 2} P_{n}^{\left(k_{2}, k_{1}\right)}(\cos 2 \varphi),
$$

where $\lambda=\left(2 n+1+k_{1}+k_{2}\right)^{2}$ and $n$ is an integer, and

$$
T(\theta)=(\cos \theta)^{k_{3}+1 / 2}(\sin \theta)^{2 n+k_{1}+k_{2}+1} P_{m}^{\left(2 n+k_{1}+k_{2}+1, k_{3}\right)}(\cos 2 \theta),
$$

where $E=\frac{1}{2}\left[2(m+n+1)+\left(k_{1}+k_{2}+k_{3}\right)\right]^{2}-\frac{1}{8}$. The orthonormal basis eigenfunctions are

$$
\Psi=(\sin \theta)^{-1} \Phi_{n}^{\left(k_{2}, k_{1}\right)}(\varphi) \Phi^{\left(2 n+k_{1}+k_{2}, k_{3}\right)}(\theta) .
$$

The operator which characterizes this solution and separation is

$$
L_{1} \Psi=\left[\left(s_{1} \frac{\partial}{\partial s_{2}}-s_{2} \frac{\partial}{\partial s_{1}}\right)^{2}+\left(\frac{1}{4}-k_{1}^{2}\right) \frac{s_{1}^{2}+s_{2}^{2}}{s_{1}^{2}}+\left(\frac{1}{4}-k_{2}^{2}\right) \frac{s_{1}^{2}+s_{2}^{2}}{s_{2}^{2}}\right] \Psi=\lambda \Psi .
$$

Note that after the multiplier $(\sin \varphi)^{k_{2}+1 / 2}(\cos \varphi)^{k_{1}+1 / 2}(\cos \theta)^{k_{3}+1 / 2}(\sin \theta)^{k_{1}+k_{2}+1}=\Pi_{\ell=1}^{3} s_{\ell}^{k_{\ell}+1 / 2}$ is split off, the eigenfunctions are polynomials in the variables $s_{2}^{2}$ and $s_{2}^{3}$. See Refs. 21-24 for a complete discussion of the polynomial setting for this eigenvalue equation.

For the second coordinate system we choose Lamé coordinates given by

$$
s_{i}^{2}=\frac{\left(u_{1}-e_{i}\right)\left(u_{2}-e_{i}\right)}{\left(e_{i}-e_{j}\right)\left(e_{i}-e_{k}\right)}, \quad i, j, k=1,2,3, \quad \text { and } i, j, k \text { pairwise distinct. }
$$

The Schrödinger equation written in terms of these coordinates is

$$
\begin{gathered}
\frac{4}{\left(u_{1}-u_{2}\right)}\left[\left(u_{1}-e_{1}\right)\left(u_{1}-e_{2}\right)\left(u_{1}-e_{3}\right)\left[\frac{\partial^{2} \Psi}{\partial u_{1}^{2}}+\frac{1}{2}\left(\frac{1}{u_{1}-e_{1}}+\frac{1}{u_{1}-e_{2}}+\frac{1}{u_{1}-e_{3}}\right) \frac{\partial \Psi}{\partial u_{1}}\right]\right. \\
\left.-\left(u_{2}-e_{1}\right)\left(u_{2}-e_{2}\right)\left(u_{2}-e_{3}\right)\left[\frac{\partial^{2} \Psi}{\partial u_{2}^{2}}-\frac{1}{2}\left(\frac{1}{u_{2}-e_{1}}+\frac{1}{u_{2}-e_{2}}+\frac{1}{u_{2}-e_{3}}\right) \frac{\partial \Psi}{\partial u_{2}}\right]\right] \\
+\left[\left(k_{1}^{2}-\frac{1}{4}\right) \frac{\left(e_{1}-e_{2}\right)\left(e_{1}-e_{3}\right)}{\left(u_{1}-e_{1}\right)\left(u_{2}-e_{1}\right)}+\left(k_{2}^{2}-\frac{1}{4}\right) \frac{\left(e_{2}-e_{1}\right)\left(e_{2}-e_{3}\right)}{\left(u_{1}-e_{2}\right)\left(u_{2}-e_{2}\right)}\right. \\
\left.+\left(k_{3}^{2}-\frac{1}{4}\right) \frac{\left(e_{3}-e_{2}\right)\left(e_{3}-e_{1}\right)}{\left(u_{1}-e_{3}\right)\left(u_{2}-e_{3}\right)}\right] \Psi=2 E \Psi .
\end{gathered}
$$

The separation equations are 


$$
\begin{gathered}
\left(\rho-e_{1}\right)\left(\rho-e_{2}\right)\left(\rho-e_{3}\right)\left[\frac{\partial^{2}}{\partial \rho^{2}}+\frac{1}{2}\left(\frac{1}{\rho-e_{1}}+\frac{1}{\rho-e_{2}}+\frac{1}{\rho-e_{3}}\right) \frac{\partial \Psi}{\partial \rho}\right]-\left[\left(k_{1}^{2}-\frac{1}{4}\right) \frac{\left(e_{1}-e_{2}\right)\left(e_{1}-e_{3}\right)}{\rho-e_{1}}\right. \\
\left.+\left(k_{2}^{2}-\frac{1}{4}\right) \frac{\left(e_{2}-e_{1}\right)\left(e_{2}-e_{3}\right)}{\rho-e_{2}}+\left(k_{3}^{2}-\frac{1}{4}\right) \frac{\left(e_{3}-e_{2}\right)\left(e_{3}-e_{1}\right)}{\rho-e_{3}}+2 E \rho+\mu\right] \Psi=0,
\end{gathered}
$$

where $\rho=u_{1}, u_{2}$. The operator whose eigenvalue is $\mu$ is

$$
\begin{aligned}
L_{2} \Psi= & \frac{-4}{\left(u_{1}-u_{2}\right)}\left[u_{2}\left[\left(u_{1}-e_{1}\right)\left(u_{1}-e_{2}\right)\left(u_{1}-e_{3}\right)\left[\frac{\partial^{2} \Psi}{\partial u_{1}^{2}}+\frac{1}{2}\left(\frac{1}{u_{1}-e_{1}}+\frac{1}{u_{1}-e_{2}}+\frac{1}{u_{1}-e_{3}}\right) \frac{\partial \Psi}{\partial u_{1}}\right]\right]\right. \\
& \left.\left.-u_{1}\left[\left(u_{2}-e_{1}\right)\left(u_{2}-e_{2}\right)\left(u_{2}-e_{3}\right)\left[\frac{\partial^{2} \Psi}{\partial u_{2}^{2}}-\frac{1}{2}\left(\frac{1}{u_{2}-e_{1}}+\frac{1}{u_{2}-e_{2}}+\frac{1}{u_{2}-e_{3}}\right) \frac{\partial \Psi}{\partial u_{2}}\right]\right]\right]\right] \\
& +\left[\left(k_{1}^{2}-\frac{1}{4}\right) \frac{\left(e_{1}-e_{2}\right)\left(e_{1}-e_{3}\right)}{\left(u_{1}-e_{1}\right)\left(u_{2}-e_{1}\right)}\left(u_{1}+u_{2}-e_{1}\right)+\left(k_{2}^{2}-\frac{1}{4}\right) \frac{\left(e_{2}-e_{1}\right)\left(e_{2}-e_{3}\right)}{\left(u_{1}-e_{2}\right)\left(u_{2}-e_{2}\right)}\right. \\
& \left.\times\left(u_{1}+u_{2}-e_{2}\right)+\left(k_{3}^{2}-\frac{1}{4}\right) \frac{\left(e_{3}-e_{2}\right)\left(e_{3}-e_{1}\right)}{\left(u_{1}-e_{3}\right)\left(u_{2}-e_{3}\right)}\left(u_{1}+u_{2}-e_{3}\right)\right] \Psi .
\end{aligned}
$$

Expressed in terms of coordinates on the sphere this has the form

$$
\begin{aligned}
L_{2}= & e_{3}\left(s_{1} \frac{\partial}{\partial s_{2}}-s_{2} \frac{\partial}{\partial s_{1}}\right)^{2}+e_{2}\left(s_{1} \frac{\partial}{\partial s_{3}}-s_{3} \frac{\partial}{\partial s_{1}}\right)^{2}+e_{1}\left(s_{3} \frac{\partial}{\partial s_{2}}-s_{2} \frac{\partial}{\partial s_{3}}\right)^{2}+\left(\frac{1}{4}-k_{1}^{2}\right)\left[\frac{e_{2} s_{2}^{2}+e_{3} s_{3}^{2}}{s_{1}^{2}}\right. \\
& \left.+e_{1}-e_{2}-e_{3}\right]+\left(\frac{1}{4}-k_{2}^{2}\right)\left[\frac{e_{1} s_{1}^{2}+e_{3} s_{3}^{2}}{s_{2}^{2}}+e_{2}-e_{1}-e_{3}\right]+\left(\frac{1}{4}-k_{3}^{2}\right)\left[\frac{e_{2} s_{2}^{2}+e_{1} s_{1}^{2}}{s_{3}^{2}}+e_{3}-e_{2}-e_{1}\right] .
\end{aligned}
$$

The eigenfunctions $\Psi$ can readily be calculated from the Schrödinger equation (67). In order to find the bound state solutions we try a solution of the form

$$
\Psi=\left(\prod_{l=1}^{3} s_{\ell}^{k_{\ell}+1 / 2}\right) \prod_{j=1}^{q}\left(\frac{s_{1}^{2}}{\theta_{j}-e_{1}}+\frac{s_{2}^{2}}{\theta_{j}-e_{2}}+\frac{s_{3}^{2}}{\theta_{j}-e_{3}}\right)
$$

(Ref. 19) and observe that

$$
\frac{s_{1}^{2}}{\theta_{j}-e_{1}}+\frac{s_{2}^{2}}{\theta_{j}-e_{2}}+\frac{s_{3}^{2}}{\theta_{j}-e_{3}}=\frac{\Pi_{\ell=1}^{2}\left(u_{\ell}-\theta_{j}\right)}{\Pi_{m=1}^{3}\left(\theta_{j}-e_{m}\right)} .
$$

For solutions, the zeros $\theta_{p}$ must satisfy the equations

$$
\frac{k_{1}+1}{\theta_{m}-e_{1}}+\frac{k_{2}+1}{\theta_{m}-e_{2}}+\frac{k_{3}+1}{\theta_{m}-e_{3}}+\sum_{j \neq m} \frac{2}{\theta_{m}-\theta_{j}}=0
$$

and the eigenvalues $E$ and $\mu$ in this case are

$$
E=\frac{1}{2}\left(2 q+2+k_{1}+k_{2}+k_{3}\right)^{2}-\frac{1}{8},
$$




$$
\begin{aligned}
\mu= & -2\left[k_{1}\left(e_{2}+e_{3}\right)+k_{2}\left(e_{1}+e_{3}\right)+k_{3}\left(e_{2}+e_{1}\right)+e_{3} k_{1} k_{2}+e_{2} k_{1} k_{3}+e_{1} k_{2} k_{3}\right] \\
& -\frac{3}{2}\left(e_{1}+e_{2}+e_{3}\right)-4 e_{2} e_{3}\left(k_{1}+1\right) \sum_{m=1}^{q} \frac{1}{\left(\theta_{m}-e_{1}\right)}-4 e_{1} e_{3}\left(k_{2}+1\right) \\
& \times \sum_{m=1}^{q} \frac{1}{\left(\theta_{m}-e_{2}\right)}-4 e_{2} e_{1}\left(k_{3}+1\right) \sum_{m=1}^{q} \frac{1}{\left(\theta_{m}-e_{3}\right)} .
\end{aligned}
$$

If we take the constants of the motion to be

$$
\begin{gathered}
H=M_{12}^{2}+M_{13}^{2}+M_{23}^{2}+\frac{k_{1}^{2}-\frac{1}{4}}{s_{1}^{2}}\left(s_{2}^{2}+s_{3}^{2}\right)+\frac{k_{2}^{2}-\frac{1}{4}}{s_{2}^{2}}\left(s_{1}^{2}+s_{3}^{2}\right)+\frac{k_{3}^{2}-\frac{1}{4}}{s_{3}^{2}}\left(s_{2}^{2}+s_{1}^{2}\right), \\
L_{1}=M_{12}^{2}+\left(k_{1}^{2}-\frac{1}{4}\right) \frac{s_{2}^{2}}{s_{1}^{2}}+\left(k_{2}^{2}-\frac{1}{4}\right) \frac{s_{1}^{2}}{s_{2}^{2}}, \\
L_{2}=e_{3} M_{12}^{2}+e_{2} M_{13}^{2}+e_{1} M_{32}^{2}+\frac{k_{1}^{2}-\frac{1}{4}}{s_{1}^{2}}\left(e_{3} s_{2}^{2}+e_{2} s_{3}^{2}\right)+\frac{k_{2}^{2}-\frac{1}{4}}{s_{2}^{2}}\left(e_{3} s_{1}^{2}+e_{1} s_{3}^{2}\right)+\frac{k_{3}^{2}-\frac{1}{4}}{s_{3}^{2}}\left(e_{1} s_{2}^{2}+e_{2} s_{1}^{2}\right),
\end{gathered}
$$

the corresponding defining relations are

$$
\begin{gathered}
L_{12}=\left\{L_{1}, L_{2}\right\}=4\left(e_{1}-e_{2}\right)\left[M_{12} M_{13} M_{32}-\left(k_{1}^{2}-\frac{1}{4}\right) \frac{s_{3} s_{2}}{s_{1}^{2}} M_{23}\right. \\
\left.-\left(k_{2}^{2}-\frac{1}{4}\right) \frac{s_{3} s_{1}}{s_{2}^{2}} M_{31}-\left(k_{3}^{2}-\frac{1}{4}\right) \frac{s_{1} s_{2}}{s_{3}^{2}} M_{12}\right], \\
L_{12}^{2}=-16 L_{1}\left(e_{2} H+\left(e_{3}-e_{2}\right) L_{1}-L_{2}\right)\left(e_{1} H+\left(e_{3}-e_{1}\right) L_{1}-L_{2}\right) \\
-16\left(k_{3}^{2}-\frac{1}{4}\right)\left(e_{1}-e_{2}\right)^{2} L_{1}^{2} \\
-16\left(k_{1}^{2}-\frac{1}{4}\right)\left(e_{2} H+\left(e_{3}-e_{2}\right) L_{1}-L_{2}\right)^{2} \\
-16\left(k_{2}^{2}-\frac{1}{4}\right)\left(e_{1} H+\left(e_{3}-e_{1}\right) L_{1}-L_{2}\right)^{2}-\frac{4}{3} \prod_{\ell=1}^{3}\left(k_{\ell}^{2}-\frac{1}{4}\right)\left(e_{1}-e_{2}\right)^{2}, \\
\left\{L_{12}, L_{1}\right\}=-\left(8 L_{1}+16\right)\left(k_{2}^{2}-\frac{1}{4}\right)\left(e_{1} H+\left(e_{3}-e_{1}\right) L_{1}-L_{2}\right) \\
\quad-\left(8 L_{1}+16\right)\left(k_{1}^{2}-\frac{1}{4}\right)\left(e_{2} H+\left(e_{3}-e_{2}\right) L_{1}-L_{2}\right),
\end{gathered}
$$




$$
\begin{aligned}
\left\{L_{12}, L_{2}\right\}= & -8\left(e_{2} H+\left(e_{3}-e_{2}\right) L_{1}-L_{2}\right)\left(e_{1} H+\left(e_{3}-e_{1}\right) L_{1}-L_{2}\right)-8\left(e_{2}-e_{3}\right) L_{1}\left(e_{1} H+\left(e_{3}\right.\right. \\
& \left.\left.-e_{1}\right) L_{1}-L_{2}\right)-8\left(e_{1}-e_{3}\right) L_{1}\left(e_{2} H+\left(e_{3}-e_{2}\right) L_{1}-L_{2}\right) \\
& +16\left(\left(k_{1}^{2}-\frac{1}{4}\right)\left(e_{3}-e_{2}\right)+\left(k_{2}^{2}-\frac{1}{4}\right)\left(e_{3}-e_{1}\right)\right) L_{2}-16\left(\left(k_{1}^{2}-\frac{1}{4}\right)\left(e_{3}-e_{2}\right)^{2}+\left(k_{2}^{2}-\frac{1}{4}\right)\right. \\
& \left.\times\left(e_{3}-e_{1}\right)^{2}+\left(k_{3}^{2}-\frac{1}{4}\right)\left(e_{1}-e_{2}\right)^{2}\right) L_{1}+16 e_{1}\left(e_{1}-e_{3}\right)\left(k_{2}^{2}-\frac{1}{4}\right)+16 e_{2}\left(e_{2}-e_{3}\right) H
\end{aligned}
$$

(ii) The second potential on the sphere is

$$
V_{2}=\frac{-\alpha s_{3}}{2 \sqrt{s_{1}^{2}+s_{2}^{2}}}+\frac{1}{4 \sqrt{s_{1}^{2}+s_{2}^{2}}}\left[\frac{k_{1}^{2}-\frac{1}{4}}{\sqrt{s_{1}^{2}+s_{2}^{2}}+s_{1}}+\frac{k_{2}^{2}-\frac{1}{4}}{\sqrt{s_{1}^{2}+s_{2}^{2}}-s_{1}}\right] .
$$

In polar coordinates the Schrödinger equation has the form

$$
\begin{aligned}
& \frac{1}{\sin \theta} \frac{\partial}{\partial \theta}\left(\sin \theta \frac{\partial \Psi}{\partial \theta}\right)+\frac{1}{\sin ^{2} \theta} \frac{\partial^{2} \Psi}{\partial \varphi^{2}}+\frac{\alpha \cos \theta}{\sin \theta} \Psi \\
& \quad+\frac{1}{2 \sin ^{2} \theta}\left(\frac{\frac{1}{4}-k_{1}^{2}}{1+\cos \varphi}+\frac{\frac{1}{4}-k_{2}^{2}}{1-\cos \varphi}\right) \Psi \\
& =-2 E \Psi .
\end{aligned}
$$

This equation can be solved by a separation of variables via the substitution $\Psi=T(\theta) \Phi(\varphi)$, and the separation equations are

$$
\begin{gathered}
\frac{\partial^{2} \Phi}{\partial \varphi^{2}}+\frac{1}{2}\left(\frac{\frac{1}{4}-k_{1}^{2}}{1+\cos \varphi}+\frac{\frac{1}{4}-k_{2}^{2}}{1-\cos \varphi}\right) \Phi=-\lambda^{2} \Phi, \\
\frac{1}{\sin \theta} \frac{\partial}{\partial \theta}\left(\sin \theta \frac{\partial T}{\partial \theta}\right)+\frac{\alpha \cos \theta}{\sin \theta} T-\frac{\lambda^{2}}{\sin ^{2} \theta} T=-2 E T .
\end{gathered}
$$

The solutions of the first equation are $\Phi_{m}^{\left(k_{1}, k_{2}\right)}(\varphi / 2)$ [see (20)], where $\lambda^{2}=\frac{1}{4}\left(2 m+k_{1}+k_{2}+1\right)^{2}$. The solution of the $\theta$ separation equation is given by

$$
\begin{aligned}
S_{n m}(\theta)= & \frac{1}{\Gamma\left(2 m+2+k_{1}+k_{2}\right)} \sqrt{\left(1+\frac{\alpha^{2}}{\left(n+m+1+\frac{1}{2}\left(k_{1}+k_{2}\right)\right)^{4}}\right) \frac{\Gamma\left(n+2(m+1)+k_{1}+k_{2}\right) \Gamma\left(i \sigma+m+1+\frac{1}{2}\left(k_{1}+k_{2}\right)\right)}{m ! \Gamma\left(i \sigma+m+\frac{1}{2}\left(k_{1}+k_{2}\right)\right)}} \\
& \times(2 \sin \theta)^{m+1+(1 / 2)\left(k_{1}+k_{2}\right)} e^{i \theta(i \sigma-n)}{ }_{2} F_{1}\left(-n, i \sigma+m+1+\frac{1}{2}\left(k_{1}+k_{2}\right) ; 2 m+2+k_{1}+k_{2} ; 1-e^{2 i \theta}\right),
\end{aligned}
$$

where $\sigma=\alpha /\left(n+m+1+\frac{1}{2}\left(k_{1}+k_{2}\right)\right)$, and the orthonormal eigenfunctions are

$$
\Psi=S_{n m}(\theta) \Phi_{m}^{\left(k_{1}, k_{2}\right)}\left(\frac{\varphi}{2}\right)
$$

The operator which characterizes the separable solutions in this coordinate system is 


$$
\begin{aligned}
L_{1} \Psi & =\left[\left(s_{1} \frac{\partial}{\partial s_{2}}-s_{2} \frac{\partial}{\partial s_{1}}\right)^{2}+\left(\frac{1}{4}-k_{1}^{2}\right) \frac{\sqrt{s_{1}^{2}+s_{2}^{2}}}{2\left(\sqrt{s_{1}^{2}+s_{2}^{2}}+s_{1}\right)}+\left(\frac{1}{4}-k_{2}^{2}\right) \frac{\sqrt{s_{1}^{2}+s_{2}^{2}}}{2\left(\sqrt{s_{1}^{2}+s_{2}^{2}}-s_{1}\right)}\right] \Psi \\
& =-\lambda^{2} \Psi .
\end{aligned}
$$

For the second coordinate system we must choose a variant of the standard elliptical coordinates. A suitable choice is

$$
s_{1}=\cos f x_{1}+\sin f x_{3}, \quad s_{2}=x_{2}, \quad s_{3}=-\sin f x_{1}+\cos f x_{3},
$$

where

$$
x_{i}^{2}=\frac{\left(y_{1}-e_{i}\right)\left(y_{2}-e_{i}\right)}{\left(e_{i}-e_{j}\right)\left(e_{i}-e_{k}\right)}, \quad i, j, k=1,2,3 \quad \text { and } i, j, k \text { pairwise distinct, } \quad \sin f=\sqrt{\frac{e_{2}-e_{1}}{e_{3}-e_{1}}} .
$$

To see how this works it is convenient to define the new variables $E_{+}, E_{-}, Z_{1}$, and $Z_{2}$ according to

$$
\begin{gathered}
e_{1}=e_{2}+\frac{1}{4}\left(E_{+}+E_{-}\right)^{2}, \quad e_{3}=e_{2}+\frac{1}{4}\left(E_{+}-E_{-}\right)^{2}, \\
y_{j}=e_{2}+\frac{1}{4}\left(E_{+}^{2}+E_{-}^{2}\right)+\frac{1}{4} E_{+} E_{-}\left(Z_{j}+\frac{1}{Z_{j}}\right) .
\end{gathered}
$$

In terms of these variables Schrödinger's equation has the form

$$
\begin{gathered}
-\frac{4 Z_{1} Z_{2}}{\left(Z_{1}-Z_{2}\right)\left(Z_{1} Z_{2}-1\right)}\left[\left(Z_{1}+\Omega_{+}\right)\left(Z_{1}+\Omega_{-}\right) Z_{1}\left[\frac{\partial^{2} \Psi}{\partial Z_{1}^{2}}+\frac{1}{2}\left(\frac{1}{Z_{1}+\Omega_{+}}+\frac{1}{Z_{1}+\Omega_{-}}+\frac{1}{Z_{1}}\right) \frac{\partial \Psi}{\partial Z_{1}}\right]\right. \\
\left.-\left(Z_{2}+\Omega_{+}\right)\left(Z_{2}+\Omega_{-}\right) Z_{2}\left[\frac{\partial^{2} \Psi}{\partial Z_{2}^{2}}+\frac{1}{2}\left(\frac{1}{Z_{2}+\Omega_{+}}+\frac{1}{Z_{2}+\Omega_{-}}+\frac{1}{Z_{2}}\right) \frac{\partial \Psi}{\partial Z_{2}}\right]\right] \\
+\left[\frac{2 i \alpha\left(Z_{1} Z_{2}+1\right)}{\left(Z_{1} Z_{2}-1\right)}+\left(k_{1}^{2}-\frac{1}{4}\right) \frac{\left(1-\Omega_{-}^{2}\right) Z_{1} Z_{2}}{\left(Z_{1} Z_{2}-1\right)\left(Z_{2}+\Omega_{+}\right)\left(Z_{1}+\Omega_{+}\right)}\right. \\
\left.+\left(k_{2}^{2}-\frac{1}{4}\right) \frac{\left(1-\Omega_{+}^{2}\right) Z_{1} Z_{2}}{\left(Z_{1} Z_{2}-1\right)\left(Z_{2}+\Omega_{-}\right)\left(Z_{1}+\Omega_{-}\right)}\right] \Psi=-2 E \Psi
\end{gathered}
$$

where $\Omega_{+}=E_{+} / E_{-}$and $\Omega_{-}=E_{-} / E_{+}$, so $\Omega_{+} \Omega_{-}=1$. The separation equations are

$$
\begin{aligned}
& 4\left(Z_{j}+\Omega_{+}\right)\left(Z_{j}+\Omega_{-}\right) Z_{j}\left[\frac{\partial^{2} \Psi}{\partial Z_{j}^{2}}+\frac{1}{2}\left(\frac{1}{Z_{j}+\Omega_{+}}+\frac{1}{Z_{j}+\Omega_{-}}+\frac{1}{Z_{j}}\right) \frac{\partial \Psi}{\partial Z_{j}}\right] \\
& +\left[2(-i \alpha+E) Z_{j}+2(i \alpha+E) \frac{1}{Z_{j}}+\frac{\left(k_{1}^{2}-\frac{1}{4}\right)\left(\Omega_{-}^{2}-1\right)}{Z_{j}+\Omega_{-}}+\frac{\left(k_{2}^{2}-\frac{1}{4}\right)\left(\Omega_{+}^{2}-1\right)}{Z_{j}+\Omega_{+}}+\mu\right] \Psi=0 .
\end{aligned}
$$

In terms of the variables $Z_{j}$ the operator which describes the separation is 


$$
\begin{aligned}
L_{2} \Psi= & \frac{4}{\left(Z_{1}-Z_{2}\right)\left(Z_{1} Z_{2}-1\right)}\left[-\left(Z_{2}+1\right) Z_{1}\left(Z_{1}+\Omega_{+}\right)\left(Z_{1}+\Omega_{-}\right)\right. \\
& \times\left[\frac{\partial^{2} \Psi}{\partial Z_{1}^{2}}+\frac{1}{2}\left(\frac{1}{Z_{1}+\Omega_{+}}+\frac{1}{Z_{1}+\Omega_{-}}+\frac{1}{Z_{1}}\right) \frac{\partial \Psi}{\partial Z_{1}}\right] \\
& \left.+\left(Z_{1}+1\right) Z_{2}\left(Z_{2}+\Omega_{+}\right)\left(Z_{2}+\Omega_{-}\right)\left[\frac{\partial^{2} \Psi}{\partial Z_{2}^{2}}+\frac{1}{2}\left(\frac{1}{Z_{2}+\Omega_{+}}+\frac{1}{Z_{2}+\Omega_{-}}+\frac{1}{Z_{2}}\right) \frac{\partial \Psi}{\partial Z_{2}}\right]\right] \\
& +\left[\frac{\left(1-\Omega_{-}^{2}\right)\left(Z_{1} Z_{2}\left(Z_{1}+Z_{2}\right)+\Omega_{-}\left(Z_{1} Z_{2}-1\right)\right)}{\left(Z_{1} Z_{2}-1\right)\left(Z_{1}+\Omega_{-}\right)\left(Z_{2}+\Omega_{-}\right)}\left(k_{1}^{2}-\frac{1}{4}\right)\right. \\
& \left.+\frac{\left(1-\Omega_{+}^{2}\right)\left(Z_{1} Z_{2}\left(Z_{1}+Z_{2}\right)+\Omega_{+}\left(Z_{1} Z_{2}-1\right)\right)}{\left(Z_{1} Z_{2}-1\right)\left(Z_{1}+\Omega_{+}\right)\left(Z_{2}+\Omega_{+}\right)}\left(k_{2}^{2}-\frac{1}{4}\right)+\frac{4 i \alpha\left(Z_{1}+Z_{2}\right)}{Z_{1} Z_{2}-1}\right] \Psi .
\end{aligned}
$$

Indeed if we consider the elliptical coordinates

$$
U_{3}^{2}=Z_{1} Z_{2}, \quad U_{1}^{2}=\frac{\left(Z_{1}+\Omega_{-}\right)\left(Z_{2}+\Omega_{-}\right)}{\Omega_{-}^{2}-1}, \quad U_{2}^{2}=\frac{\left(Z_{1}+\Omega_{+}\right)\left(Z_{2}+\Omega_{+}\right)}{\Omega_{+}^{2}-1},
$$

then putting $k_{3}=\sqrt{\frac{1}{4}+2(E-i \alpha)}$ and $\widetilde{E}=i \alpha+E$ and multiplying the Schrödinger equation by $\left(1 / Z_{1} Z_{2}-1\right)$ we see that the resulting equation has the form

$$
\begin{aligned}
H \Psi= & \frac{\partial^{2} \Psi}{\partial U_{1}^{2}}+\frac{\partial^{2} \Psi}{\partial U_{2}^{2}}+\frac{\partial^{2} \Psi}{\partial U_{3}^{2}}-\left(U_{1} \frac{\partial}{\partial U_{1}}+U_{2} \frac{\partial}{\partial U_{2}}+U_{3} \frac{\partial}{\partial U_{3}}\right)^{2} \Psi \\
& -\left(U_{1} \frac{\partial \Psi}{\partial U_{1}}+U_{2} \frac{\partial \Psi}{\partial U_{2}}+U_{3} \frac{\partial \Psi}{\partial U_{3}}\right)+\left[\frac{\frac{1}{4}-k_{1}^{2}}{U_{1}^{2}}+\frac{\frac{1}{4}-k_{2}^{2}}{U_{2}^{2}}+\frac{\frac{1}{4}-k_{3}^{2}}{U_{3}^{2}}\right] \Psi+2 \widetilde{E} \Psi=0 .
\end{aligned}
$$

We recognize this as Eq. (67) that we obtained in the case of the potential $V_{1}$. Thus the solutions are of the form

$$
\Psi=\left(\prod_{\ell=1}^{3} U_{\ell}^{k /+1 / 2}\right) \prod_{j=1}^{q}\left(\frac{U_{1}^{2}}{\theta_{j}-e_{1}}+\frac{U_{2}^{2}}{\theta_{j}-e_{2}}+\frac{U_{3}^{2}}{\theta_{j}-e_{3}}\right) .
$$

The zeros $\theta_{p}$ satisfy the equations

$$
\frac{k_{1}+1}{\theta_{m}+\Omega_{-}}+\frac{k_{2}+1}{\theta_{m}+\Omega_{+}}+\frac{\sqrt{\frac{1}{4}+2(E-i \alpha)}+1}{\theta_{m}}+\sum_{j \neq m} \frac{2}{\theta_{m}-\theta_{j}}=0
$$

and the eigenvalues $E$ and $\mu$ are determined by 


$$
\begin{gathered}
E=\frac{1}{2}\left(2 q+2+k_{1}+k_{2}+\sqrt{\frac{1}{4}+2(E-i \alpha)}\right)^{2}-\frac{1}{8}, \\
\mu=2\left[\Omega_{-}\left(k_{2}+\sqrt{\frac{1}{4}+2(E-i \alpha)}\right)+\Omega_{+}\left(k_{1}+\sqrt{\frac{1}{4}+2(E-i \alpha)}\right)\right] \\
+2\left[\Omega_{+} k_{1}+\Omega_{-} k_{2}\right] \sqrt{\frac{1}{4}+2(E-i \alpha)}+\frac{3}{2}\left(\Omega_{+}+\Omega_{-}\right)+\sqrt{\frac{1}{4}+2(E-i \alpha)} \\
\times\left[4 \Omega_{+}\left(k_{1}+1\right) \sum_{m=1}^{q} \frac{1}{\left(\theta_{m}+\Omega_{-}\right)}+4 \Omega_{-}\left(k_{2}+1\right) \sum_{m=1}^{q} \frac{1}{\left(\theta_{m}+\Omega_{+}\right)}\right] \\
-4 \Omega_{+} \Omega_{-}\left(\sqrt{\frac{1}{4}+2(E-i \alpha)}+1\right) \sum_{m=1}^{q} \frac{1}{\theta_{m}} .
\end{gathered}
$$

The classical constants of the motion associated with this potential are

$$
\begin{aligned}
H= & -\frac{4 Z_{1} Z_{2}}{\left(Z_{1}-Z_{2}\right)\left(Z_{1} Z_{2}-1\right)}\left[\left(Z_{1}+\Omega_{+}\right)\left(Z_{1}+\Omega_{-}\right) Z_{1} P_{Z_{1}}^{2}-\left(Z_{2}+\Omega_{+}\right)\left(Z_{2}+\Omega_{-}\right) Z_{2} P_{Z_{2}}^{2}\right] \\
& +\left[\frac{2 i \alpha\left(Z_{1} Z_{2}+1\right)}{\left(Z_{1} Z_{2}-1\right)}+\left(k_{1}^{2}-\frac{1}{4}\right) \frac{\left(1-\Omega_{-}^{2}\right) Z_{1} Z_{2}}{\left(Z_{1} Z_{2}-1\right)\left(Z_{2}+\Omega_{+}\right)\left(Z_{1}+\Omega_{+}\right)}\right. \\
& \left.+\left(k_{2}^{2}-\frac{1}{4}\right) \frac{\left(1-\Omega_{+}^{2}\right) Z_{1} Z_{2}}{\left(Z_{1} Z_{2}-1\right)\left(Z_{2}+\Omega_{-}\right)\left(Z_{1}+\Omega_{-}\right)}\right], \\
L_{2}= & \frac{4}{\left(Z_{1}-Z_{2}\right)\left(Z_{1} Z_{2}-1\right)}\left[-\left(Z_{2}+1\right) Z_{1}\left(Z_{1}+\Omega_{+}\right)\left(Z_{1}+\Omega_{-}\right) P_{Z_{1}}^{2}\right. \\
& \left.+\left(Z_{1}+1\right) Z_{2}\left(Z_{2}+\Omega_{+}\right)\left(Z_{2}+\Omega_{-}\right) P_{Z_{2}}^{2}\right] \\
& +\frac{\left(1-\Omega_{-}^{2}\right)\left(Z_{1} Z_{2}\left(Z_{1}+Z_{2}\right)+\Omega_{-}\left(Z_{1} Z_{2}-1\right)\right)}{\left(Z_{1} Z_{2}-1\right)\left(Z_{1}+\Omega_{-}\right)\left(Z_{2}+\Omega_{-}\right)}\left(k_{1}^{2}-\frac{1}{4}\right) \\
& +\frac{\left(1-\Omega_{+}^{2}\right)\left(Z_{1} Z_{2}\left(Z_{1}+Z_{2}\right)+\Omega_{+}\left(Z_{1} Z_{2}-1\right)\right)}{\left(Z_{1} Z_{2}-1\right)\left(Z_{1}+\Omega_{+}\right)\left(Z_{2}+\Omega_{+}\right)}\left(k_{2}^{2}-\frac{1}{4}\right)+\frac{4 i \alpha\left(Z_{1}+Z_{2}\right)}{Z_{1} Z_{2}-1} \\
= & \left(\Omega_{+}+\Omega_{-}\right)\left(M_{12}^{2}-M_{13}^{2}-M_{23}^{2}\right)-2 i\left(\Omega_{-}-\Omega_{+}\right) M_{12} M_{13}+\left[\frac{\Omega_{+}\left(s_{3}-i s_{1}\right)+\Omega_{-}\left(s_{3}+i s_{1}\right)}{\sqrt{s_{1}^{2}+s_{2}^{2}}}\right] \alpha \\
& +\frac{k_{1}^{2}-\frac{1}{4}}{2\left(\sqrt{s_{1}^{2}+s_{2}^{2}}+s_{1}\right)}\left[\frac{\Omega_{+}\left(s_{3}-i s_{1}\right)+\Omega_{-}\left(s_{3}+i s_{1}\right)}{\sqrt{s_{1}^{2}+s_{2}^{2}}\left(\sqrt{s_{1}^{2}+s_{2}^{2}}-s_{3}\right)}+\Omega_{-}\left(\sqrt{s_{1}^{2}+s_{2}^{2}}+i s_{3}\right)\right] \\
& \frac{k_{2}^{2}-\frac{1}{4}}{2\left(\sqrt{\left.s_{1}^{2}+s_{2}^{2}-s_{1}\right)}\right.}\left[\frac{\Omega_{+}\left(s_{3}-i s_{1}\right)+\Omega_{-}\left(s_{3}+i s_{1}\right)}{\sqrt{s_{1}^{2}+s_{2}^{2}}\left(\sqrt{s_{1}^{2}+s_{2}^{2}}-s_{3}\right)}+\Omega_{+}\left(\sqrt{s_{1}^{2}+s_{2}^{2}}+i s_{3}\right)\right], \\
&
\end{aligned}
$$




$$
\begin{aligned}
L_{1}= & -\frac{\left(Z_{1}+\Omega_{+}\right)\left(Z_{1}+\Omega_{-}\right)\left(Z_{2}+\Omega_{+}\right)\left(Z_{2}+\Omega_{-}\right)}{\left(Z_{1}-Z_{2}\right)^{2}}\left(Z_{1} P_{Z_{1}}-Z_{2} P_{Z_{2}}\right)^{2} \\
& +\frac{1}{4}\left(k_{2}^{2}-\frac{1}{4}\right) \frac{\left(\Omega_{+}^{2}-1\right)\left(Z_{1} Z_{2}-1\right)}{\left(Z_{1}+\Omega_{+}\right)\left(Z_{2}+\Omega_{+}\right)}+\frac{1}{4}\left(k_{1}^{2}-\frac{1}{4}\right) \frac{\left(\Omega_{-}^{2}-1\right)\left(Z_{1} Z_{2}-1\right)}{\left(Z_{1}+\Omega_{-}\right)\left(Z_{2}+\Omega_{-}\right)} \\
= & M_{12}^{2}-\frac{1}{2}\left[\frac{\sqrt{s_{1}^{2}+s_{2}^{2}}}{\sqrt{s_{1}^{2}+s_{2}^{2}+s_{1}}}\left(k_{1}^{2}-\frac{1}{4}\right)+\frac{\sqrt{s_{1}^{2}+s_{2}^{2}}}{\sqrt{s_{1}^{2}+s_{2}^{2}}-s_{1}}\left(k_{2}^{2}-\frac{1}{4}\right)\right] .
\end{aligned}
$$

The corresponding defining relations for the quadratic algebra are

$$
\begin{aligned}
& L_{12}^{2}=-64 L_{1}^{2}-4\left(\Omega_{+}+\Omega_{-}\right)^{2} H^{2} L_{1}+64 H L_{2}^{2}+16\left(\Omega_{+}+\Omega_{-}\right) L_{3} L_{2}^{2}-4 L_{1} L_{2}^{2}-8\left(\Omega_{+}+\Omega_{-}\right) L_{1} L_{2} H \\
& +\left(\Omega_{+}-\Omega_{-}\right)-4\left(\Omega_{-}\left(4 k_{1}^{2}-1\right)-\Omega_{+}\left(4 k_{2}^{2}-1\right)\right) L_{1}^{2}-4\left(\Omega_{-}\left(1-k_{2}^{2}-3 k_{1}^{2}\right)\right. \\
& \left.-\Omega_{+}\left(1-k_{1}^{2}-3 k_{2}^{2}\right)\right) H L_{1}+4\left(k_{1}^{2}-k_{2}^{2}\right) L_{1} L_{2}-4 i \alpha\left(k_{1}^{2}-k_{2}^{2}\right) L_{2}+8 i \alpha\left(\Omega_{-}\left(k_{2}^{2}-k_{1}^{2}-2 i \alpha\right)\right. \\
& \left.+\Omega_{+}\left(k_{1}^{2}-k_{2}^{2}+2 i \alpha\right)\right) L_{1}-\left(k_{1}^{2}-k_{2}^{2}\right)\left(\Omega_{-}\left(k_{2}^{2}-k_{1}^{2}+4 i \alpha\right)+\Omega_{+}\left(k_{1}^{2}-k_{2}^{2}+4 i \alpha\right)\right) H \\
& +2 i \alpha\left(\Omega_{+}-\Omega_{-}\right)\left(\left(k_{1}^{2}-k_{2}^{2}\right)^{2}+2 i \alpha\left(2\left(k_{1}^{2}+k_{2}^{2}\right)-1\right)\right), \\
& \left\{L_{12}, L_{1}\right\}=8\left(\Omega_{+}+\Omega_{-}\right) L_{1}^{2}-4 L_{1} L_{2}-4\left(\Omega_{+}+\Omega_{-}\right) L_{1} H+2\left(k_{1}^{2}-k_{2}^{2}\right) L_{1} \\
& \quad-2 i \alpha\left(k_{1}^{2}-k_{2}^{2}\right)\left(\Omega_{+}-\Omega_{-}\right), \\
& \left\{L_{12}, L_{2}\right\}=-2\left(\Omega_{+}+\Omega_{-}\right)^{2} H^{2}-96 L_{1}^{2}-2 L_{2}^{2}+16 L_{2} L_{1}-4\left(\Omega_{+}+\Omega_{-}\right) L_{2} H-2 L_{1} H\left(\Omega_{+}-\Omega_{-}\right) \\
& \quad \times\left[\Omega_{+}\left(k_{1}^{2}+3 k_{2}^{2}-1\right)-\Omega_{-}\left(k_{2}^{2}+3 k_{1}^{2}-1\right)\right] H-4\left(\Omega_{+}\left(1-4 k_{2}^{2}\right)-\Omega_{+}\left(1-4 k_{2}^{2}\right)\right) L_{1} \\
& +4 i \alpha\left(\left(k_{1}^{2}-k_{2}^{2}\right)\left(\Omega_{+}-\Omega_{-}\right)+2 i \alpha\left(\Omega_{+}-\Omega_{-}\right)\right) .
\end{aligned}
$$

\section{THE $n$-DIMENSIONAL ISOTROPIC OSCILLATOR}

It is well known that the quantum isotropic harmonic oscillator can be solved by the method of separation of variables in a number of coordinate systems, including Cartesian coordinates and elliptical coordinates (see, e.g., Refs. 25 and 26). We present a new derivation of the eigenfunctions associated with elliptic coordinates. We will adopt the convention of defining this equation by

$$
H \Psi=\left[-\frac{1}{2} \Delta+\frac{1}{2} \omega^{2}\left(x_{1}^{2}+\cdots+x_{n}^{2}\right)\right] \Psi=E \Psi,
$$

where $\Delta=\partial_{x_{1}}^{2}+\cdots+\partial_{x_{n}}^{2}$. Elliptical coordinates in Euclidean $n$ space are defined by

$$
x_{j}^{2}=\frac{\Pi_{\ell=1}^{n}\left(u_{\ell}-e_{j}\right)}{\Pi_{k \neq j}\left(e_{k}-e_{j}\right)}
$$

for $e_{1}<u_{1}<e_{2}<\cdots<e_{n}<u_{n}$. To obtain polynomial solutions of this equation in these coordinates we note the identity

$$
\sum_{k=1}^{n} \frac{x_{k}^{2}}{\theta-e_{k}}-1=-\frac{\Pi_{h=1}^{n}\left(\theta-u_{h}\right)}{\Pi_{\ell=1}^{n}\left(\theta-e_{\ell}\right)} .
$$

If we rewrite Eq. (87) in terms of $\Phi$ where $\Psi=\exp \left[-1 / 2 \omega^{2} r^{2}\right] \Phi$ and $r$ is the radial coordinate, we obtain the eigenvalue equation 


$$
\left[\Delta-2 \omega\left(\sum_{m=1}^{n} x_{m} \partial_{x_{m}}\right)-n \omega\right] \Phi=-2 E \Phi .
$$

Note that the operator on the left-hand side maps polynomials in the variables $x_{j}$ to polynomials, without increasing the order. For this equation we look for solutions of the form

$$
\Phi=\prod_{\ell=1}^{q} x_{\ell}^{\alpha} \zeta\left(x_{1}, \ldots, x_{n}\right)
$$

where $\alpha_{\ell}=0$ or 1 . The equation for $\zeta$ is then

$$
\left[\Delta+\sum_{m=1}^{n}\left(\frac{2 \alpha_{m}}{x_{m}} \partial_{x_{m}}-2 \omega x_{m} \partial_{x_{m}}\right)\right] \zeta=\left(-2 E+n \omega+2 \omega \sum_{\ell=1}^{n} \alpha_{\ell}\right) \zeta
$$

We now look for solutions of the form

$$
\zeta=\prod_{m=1}^{q}\left[\sum_{k=1}^{n} \frac{x_{k}^{2}}{\theta_{m}-e_{k}}-1\right]
$$

Substituting this ansatz into the equation for $\zeta$ we obtain the requirements

$$
\sum_{k=1}^{n} \frac{1+2 \alpha_{k}}{\theta_{\ell}-e_{k}}+\sum_{j \neq \ell} \frac{4}{\theta_{\ell}-\theta_{j}}=-2 \omega, \quad E=\omega\left(2 m+\frac{n}{2}+\sum_{p=1}^{n} \alpha_{p}\right) .
$$

The operators which describe the separation constants are

$$
\Lambda_{j}=\sum_{k \neq \ell} S_{j-1}^{k \ell} M_{k l}^{2}+\sum_{\ell \neq j} S_{j}^{\ell} Q_{\ell \ell}, \quad j=1, \ldots, n-1,
$$

where $M_{k \ell}=x_{k} \partial_{x_{\ell}}-x_{\ell} \partial_{x_{k}}$, and

$$
Q_{i k}=\partial_{x_{i}} \partial_{x_{k}}-\omega^{2} x_{i} x_{k}, \quad S_{j}^{k l}=\frac{1}{j !} \sum_{i_{1}, \ldots, i_{j}} e_{i_{1}} \cdots e_{i_{j}},
$$

where $i_{m} \neq i_{m^{\prime}}, i_{m} \neq k, l$ for $m \neq m^{\prime}$ and $m, m^{\prime}=1 \cdots j$, and

$$
S_{j}^{k}=S_{j}^{k}\left[e_{1}, \ldots, e_{n}\right]=\frac{1}{j !} \sum_{i_{1}, \ldots, i_{j}} e_{i_{1}} \cdots e_{i_{j}}, \quad i_{1}, \ldots, i_{j} \text { pairwise distinct, }
$$

where $i_{m} \neq i_{m^{\prime}}, i_{m} \neq k$ for $m \neq m^{\prime}$ and $m, m^{\prime}=1 \cdots j$. In coordinate form these operators are

$$
\Lambda_{j}=\sum_{k=1}^{n} \frac{S_{j}^{k}\left[u_{1}, \ldots, u_{n}\right]}{\Pi_{\ell \neq k}\left(u_{\ell}-u_{k}\right)}\left(4 \sqrt{\Pi_{\ell=1}^{n}\left(u_{k}-e_{\ell}\right)} \partial_{u_{k}} \sqrt{\Pi_{\ell=1}^{n}\left(u_{k}-e_{\ell}\right)} \partial_{u_{k}}-\omega^{2} \Pi_{\ell=1}^{n}\left(u_{k}-e_{\ell}\right)\right) .
$$

They have the eigenvalues

$$
\sum S_{j}^{\ell}\left[\omega\left(2 \alpha_{\ell}+1\right)-\sum_{m=1}^{q} \frac{2\left(\alpha_{\ell}+1\right)}{\theta_{m}-e_{\ell}}\right] .
$$

These operators form a closed algebra under the commutation relations 


$$
\begin{gathered}
{\left[M_{k l}, M_{i j}\right]=M_{l j} \delta_{k i}+M_{k i} \delta_{l j}-M_{k j} \delta_{l i}-M_{l i} \delta_{k j},} \\
{\left[Q_{i k}, Q_{j l}\right]=\omega^{2}\left[M_{i j} \delta_{l k}+M_{i l} \delta_{j k}+M_{k j} \delta_{l i}+M_{k l} \delta_{j i}\right],} \\
{\left[Q_{i k}, M_{j l}\right]=Q_{i j} \delta_{k l}+Q_{k j} \delta_{i l}+Q_{i l} \delta_{k j}+Q_{k l} \delta_{i j} .}
\end{gathered}
$$

The eigenfunctions can be normalized by using their representation in terms of Cartesian coordinates. For the assumed form of the eigenfunctions given above the norm $N$ is readily calculated to be

$$
\begin{aligned}
& N^{2}=\sum_{p=0}^{n} \sum_{s_{i_{1}} s_{i_{2}}, \ldots, s_{i_{p}}} \sum_{i_{1}, \ldots, i_{p} \neq}(-1)^{q} \omega^{-\left(n / 2+\Sigma_{h=1}^{n} \alpha_{h}\right)}
\end{aligned}
$$

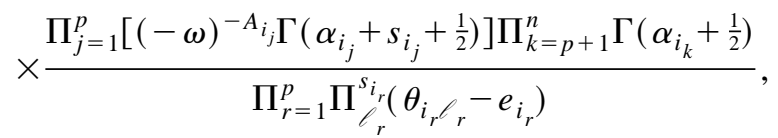

where $0 \leqslant s_{i_{j}} \leqslant 2 q, 0 \leqslant \sum_{j=1}^{p} s_{i_{j}} \leqslant 2 q, 0 \leqslant p \leqslant n$. Each of the $s_{i_{j}}$ are integers, $\theta_{i_{r}} \ell_{r} \in\left\{\theta_{1}, \ldots, \theta_{m}\right\}$ and $\theta_{i_{r} \ell} \neq \theta_{j_{w} \ell_{w}}$ if $i_{r} \neq j_{w}$, and if $i_{r}$ is fixed then at most two $\theta_{i_{r} \ell}$ can be equal. From these results we have the satisfactory situation that both the eigenvalues and the normalizations can be computed in terms of the zeros $\theta_{1}, \ldots, \theta_{m}$.

As an example of the simplest normalized eigenfunction we give the following:

$$
\begin{aligned}
\Psi= & \sqrt{\frac{\omega}{\pi}}\left[\frac{3}{4 \omega^{2}}\left(\frac{1}{\left(\theta-e_{1}\right)^{2}}+\frac{1}{\left(\theta-e_{2}\right)^{2}}\right)+\frac{1}{2 \omega^{2}\left(\theta-e_{1}\right)\left(\theta-e_{2}\right)}+1\right]^{-1 / 2} \\
& \times e^{-\left(x^{2}+y^{2}\right) / 2}\left[\frac{x^{2}}{\theta-e_{1}}+\frac{y^{2}}{\theta-e_{2}}-1\right] .
\end{aligned}
$$

For this eigenfunction the varable $\theta$ is a solution of

$$
\frac{1}{\left(\theta-e_{1}\right)}+\frac{1}{\left(\theta-e_{2}\right)}=0
$$

i.e., $\theta=\left(e_{1}+e_{2}\right) / 2$. If we substitute this into the above equations we obtain

$$
\Psi=\sqrt{\frac{\omega}{\pi}}\left[\left(\frac{2}{\omega\left(e_{1}-e_{2}\right)}\right)^{2}+1\right]^{-1 / 2} e^{-\omega\left(x^{2}+y^{2}\right) / 2}\left[\frac{2\left(x^{2}-y^{2}\right)}{\left(e_{2}-e_{1}\right)}-1\right] .
$$

The orthonormalized eigenfunctions calculated in this way form a complete set.

In this paper we have established that once the zeros of a given set of special eigenfunctions, which are essentially polynomials, are known, then all the other properties such as normalization and eigenvalues can be determined from them in terms of algebraic expressions.

\section{ACKNOWLEDGMENT}

W.M. was supported in part by the National Science Foundation under Grant No. DMS 94-00533.

${ }^{1}$ L. P. Eisenhart, “Enumeration of Potentials for which One-Particle Schrödinger Equations are Separable,' Phys. Rev. 74, 87 (1948).

${ }^{2}$ J. Frišs, V. Mandrosov, Ya. A. Smorodinsky, M. Uhlir, and P. Winternitz, ' On Higher Symmetries in Quantum Mechanics," Phys. Lett. 16, 354 (1965). 
${ }^{3}$ J. Friš, Ya. A. Smorodinskii, M. Uhlír, and P. Winternitz, "Symmetry Groups in Classical and Quantum Mechanics," Sov. J. Nucl. Phys. 4, 444 (1967).

${ }^{4}$ A. A. Makarov, Ya. A. Smorodinsky, Kh. Valiev, and P. Winternitz, "A Systematic Search for Nonrelativistic Systems with Dynamical Symmetries," Nuovo Cimento A 52, 1061 (1967).

${ }^{5}$ D. Bonatos, C. Daskaloyannis, and K. Kokkotas, "Deformed Oscillator Algebras for Two-Dimensional Quantum Superintegrable Systems," Phys. Rev. A 50, 3700 (1994).

${ }^{6}$ F. Calogero, "Solution of a three-body problem in one dimension," J. Math. Phys. 10, 2191 (1969).

${ }^{7}$ A. Cisneros and H. V. McIntosh, "Symmetry of the two-dimensional hydrogen atom," J. Math. Phys. 10, 277 (1969).

${ }^{8}$ L. G. Mardoyan, G. S. Pogosyan, A. N. Sissakian, and V. M. Ter-Antonyan, "Elliptic Basis for a Circular Oscillator," Nuovo Cimento B 88, 43 (1985); “'Two-Dimensional Hydrogen Atom: I. Elliptic Bases,” Theor. Math. Phys. 61, 1021 (1984); "Hidden symmetry, Separation of Variables and Interbasis Expansions in the Two-Dimensional Hydrogen Atom," J. Phys. A 18, 455 (1985).

${ }^{9}$ B. Zaslow and M. E. Zandler, "Two-dimensional analog of the hydrogen atom," Am. J. Phys. 35, 1118 (1967).

${ }^{10}$ N. W. Evans, "Superintegrability in Classical Mechanics," Phys. Rev. A 41, 5666 (1990); "Group theory of the Smorodinsky-Winternitz system," J. Math. Phys. 32, 3369 (1991).

${ }^{11}$ N. W. Evans, "Superintegrability of the Calogero-Moser System," Phys. Lett. A 95, 279 (1983); “'Super-Integrability of the Winternitz System," Phys. Lett. A 147, 483 (1990).

${ }^{12}$ H. L. Krall and I. M. Sheffer, “'Orthogonal Polynomials in Two Variables,” Ann. Mat. Pura Appl. 76(4), 325 (1967).

${ }^{13}$ W. Miller, Jr., Symmetry and Separation of Variables (Addison-Wesley, Providence, RI, 1977).

${ }^{14}$ Ya. A. Granovsky, A. S. Zhedanov, and I. M. Lutzenko, “Quadratic Algebra as a 'Hidden' Symmetry of the Hartmann Potential," J. Phys. A 24, 3887 (1991).

${ }^{15}$ M. Kibler and T. Negadi, Creatica Chemica Acta CCACCA, 57(6), 1509-1523 (1984).

${ }^{16}$ C. Grosche, G. S. Pogosyan, and A. N. Sissakian, "Path Integral Discussion for Smorodinsky-Winternitz Potentials: I. Two- and Three-Dimensional Euclidean Space,"' Fortschr. Phys. 43, 453 (1995).

${ }^{17} \mathrm{C}$. Boyer, "Lie Theory and Separation of Variables for the Equation $i U_{t}+\Delta_{2} U-\left(\alpha / x_{1}^{2}+\beta / x_{2}^{2}\right) U=0$," SIAM J. Math. Anal. 7, 230 (1976).

${ }^{18}$ P. Letourneau and L. Vinet, "Superintegrable Systems: Polynomial Algebras and Quasi-Exactly Solvable Hamiltonians," Ann. Phys. 243, 144 (1995).

${ }^{19}$ E. T. Whittaker and G. N. Watson, A Course of Modern Analysis, Vol. II (Cambridge U.P., Cambridge, 1927).

${ }^{20}$ C. R. Holt, "Construction of new integrable Hamiltonians in two degrees of freedom," J. Math. Phys. 23, 1037 (1982).

${ }^{21}$ E. G. Kalnins, W. Miller, and M. Tratnik, "Families of Orthogonal and Biorthogonal Polynomials on the $n$-sphere," SIAM J. Math. Anal. 22, 272 (1991).

${ }^{22}$ E. G. Kalnins and W. Miller, "Hypergeometric Expansions of Heun Polynomials," SIAM J. Math. Anal. 22, 1450 (1991).

${ }^{23}$ E. G. Kalnins and W. Miller, "Orthogonal Polynomials on $n$-spheres: Gegenbauer, Jacobi and Heun,” in Topics in Polynomials of One and Several Variables and their Applications: A Legacy of P. L. Chebyshev (1821-1894), edited by H. M. Srivastava, T. M. Rassias, and A. Yanushauskas (World Scientific, Singapore, 1991).

${ }^{24}$ J. Patera and P. Winternitz, "A New basis for the representation of the rotation group. Lamé and Heun polynomials," J. Math. Phys. 14, 1130 (1973).

${ }^{25}$ C. Boyer, E. Kalnins, and W. Miller, Jr., "Lie theory and separation of variables, 7; The Harmonic Oscillator in Elliptic Coordinates and Ince Polynomials," J. Math. Phys. 16, 512 (1975).

${ }^{26}$ W. Kallies, I. Lukach, G. S. Pogosyan, and A. N. Sissakian, "Ellipsoidal Bases for Harmonic Oscillator,' in Proceedings of the "International Workshop on 'Symmetry Methods in Physics' in Memory of Prof. Ya. A. Smorodinsky", (Publishing Department of JINR, Dubna, 1994), Vol. 1, p. 206, Preprint JINR, E2-94-230, Dubna, 1994. 